8 
QL $=\quad 598,28$

675

A $16 X$

1853

Birds

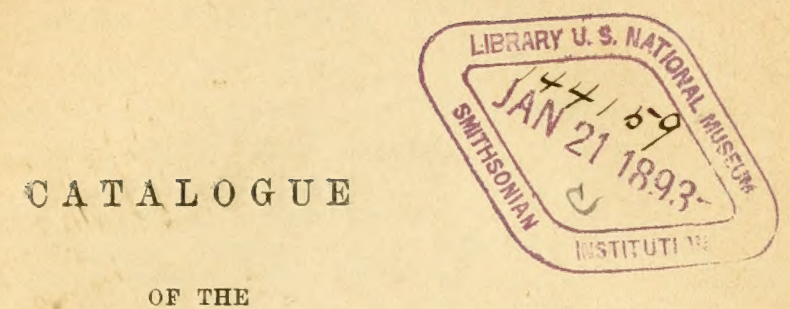

00LOGICAL COLLECTION

IN

\section{THE ACADEMY OF NATURAL SCIENCES}

OF PHILADELPHIA:

BY

A. L. HEER M A N, M. D

MARCH 1, 1853. 



\section{1st Order ACCIPITRES.}

\section{Family VULTURID虑.}

Grpaetus barbatus (Linn.) Greece. From Mons. Des Murs' Collection. Nrophron percnopterus (Linn.) France. From the same.

Cathartes iota (Molina.) Buenos Ayres. From the same. " Brasiliensis (Lath.) Brazil. From the same. " Aura (Linn.) Maryland. Presented by A. L. Heermann, M. D. " atratus (Bartr.) S. Carolina. From the same.

Vultur monachus Lirn. Greece. From Mons. Des Murs' Collection.

$\begin{array}{ccc}\text { Otogyps auricularis (Daud.) } & \text { Africa. } & \text { From the same. } \\ \text { Gyps Fulvus (Gmel.) } & \begin{array}{l}\text { France. } \\ \text { Greece. }\end{array} & \text { From the same. } \\ \text { "6 } & \text { From the same. }\end{array}$

\section{Family FALCONID Æ.}

Milvago chimango (Vieill.) Paraguay. From Mons. Des Murs' Collection.

Polyborus vulgaris Vieill. Paraguay. From the same.
"
“
Cuba. From Mons. La Sagra's Collection.

Buteo vulgaris Bechst. France. From Mons. Des Murs' Collection.

" Jackal (Daud.) Africa. From the same.

"6 ERXthronotus (King.) Chili. From the same.

"6 Rutilans (Lichst.) Paraguay. From the same.

"6 Borealis (Gmel.) N. America. From the same.

" Lineatus (Gmel.) Pennsylvania. Presented by Mr. John Krider.

Archibuteo Lagopus (Brün.) France. From Mons. Des Murs' Collection.

Aquila fulva (Linn.) France, Pyrenees. From the same.

Switzerland. Presented by A. L. Heermann, M. D.

"6 Heliaca Sav. Algeria. From Mons. Des Murs' Collection.

" Nevia (Gmel.) Island of Rugen. From the same.

"Bonelin (Temm.) Greece. From the same.

" Morphuoldes Gould. Australia. From Mr. J. Gould's Collection.

"s Audax (Lath.) Africa. Presented by T. B. Wilson, M. D.

Circaetus gallicus (Gmel.) France. From Mons. Des Murs' Collection. "6 Thoracicus Cuvier. Africa. From the same.

Pandion halietes (Linn.) France. From the same.

" Carolinensis (Gmel.) New Jersey. Presented by Mr. S. Ashmead.

" " $"$ N. Jersey. Presented by Wm. Gambel, M.D.

6 " 6 Maryland. Presented by A. L. Heermann, M.D

"G Levcocephalus Gould. Australia. From Mr. J. Gould's Collection.

Halizetus albicillus Linn. Europe. From Mons. Des Murs' Collection.

"6 Leucocephalus (Linn.) N. Jersey. Presented by Mr. S. Ashmead,

" Macei (Temm.) India. From Mons. Des Murs' Collectios.

" vocifer (Daud.) South Africa. From the same.

Helotarsi's ecaudates (Shaw.) South Africa. From the same. 
Haliastur Leucostemus (Gould.) Australia. From Mr. J. Gould's Collection. " Sphenurus (Vieill.) Australia. Ditto.

" Indus (Bodd.) India. Presented by T. B. Wilson, M. D.

Falco gyrfalco Linn. Iceland. From Mons. Des Murs' Collection.

" PEREGrinus Linn. France. From the same.

" " Saxony. From the same.

" " G Great Britain. From the same.

6. Melanogenys Gould. Australia. From Mr. J. Gould's Collection.

Hypotriorchis subbuteo (Linn.) France. From Mons Des Murs'Collection. "6 "Germany. From the same.

" TESALON (Gmel.) France. From the same.

"6 Eleanore (Gené.) Italy. From the same.

" " Algiers. Presented by Mr. E. Wilson.

" Frontatus (Gould.) Australia. From Mr.J. Gould's Collection.

Jeracidea ferigora (Vigs. and Horsf.) Australia. From the same.

" occidentalis Gould. Australia. From the same.

Tinnuncudess alaudarius (Briss.) West Africa. From Mons. Des Murs? Collection.

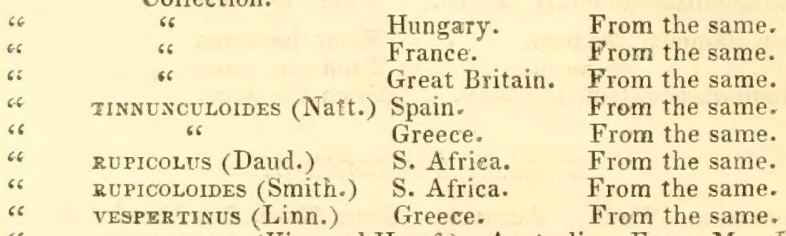

Cenchromes (Vig. and Horsf.) Australia. From Mr. J. Goald's Collection.

"s Sparverius (Linn.) Cuba. From Mons. La Sagra's Colleetion.

Pernis apivorus (Lirn.) France. From Mons. Des Mars' Collection.

Mrluves regalis Briss. France. From the same.

" ATER (Gmel.) Greece. From the same.

" "W. Africa. From the same.

" Isurus Gould. Australia. From Mr. J. Gould's Collection.

" Cheela (Lath.) India. Presented by Mr. E. Wilson.

Elanus melanopterus (Daud.) Egypt. From Mons. Des Murs' Collection.

Astur palumbarius (Linn.) France. From the same.

" Nove Holdandie (Gmel.) Australia. From the same.

" atricapillus (Wilson.) Mass. Presented by T. B. Wilson, M. D.

Accipiter nistrs (Linn.) France. From Mons. Des Murs' Collection.

" RUFIVENTRis Smith. S. Africa. From the same.

" Fuscus (Gmel.) N. Jersey. Presented by Mr. J. Krider.

" Cooperii (Pr. Bonap.) Penn. Presented by C. Pickering, M. D.

"G Cruentus (Gould.) Australia. From Mr. J. Gould's Collection.

"6 ApProximans (Vig. and Horsf.) Australia. From the same.

Melierax Canorus (Thunb.) S. Africa. From Mons. Des Murs' Colleetion.

Serpentarius reptilivorus Daud. S. Africa. From the same.

Circus cyaneus (Linn.) France. From the same.

" Frruginosus (Linn.) France. From the same.

" cinerascens (Mont.) France. From the same.

" vliginosus (Gmel.) Missouri. Presented by A. L. Heermann, M. D.

$\because \quad$ Assimilis Jard. and Selby. Australia. From Mr. J. Gould's Collection.

"J Jardini Gould. Australia. From the same.

"s Rakivorus (Daud.) S. Africa. Presented by Mr. E. Wilson.

${ }^{6}$ araunes (Temm.) Tangier. From Mons. Des Murs' Collection. 
III. Family STRIGID Æ.

Strix flammea Linn. France. From Mons. Des Murs' Collection.
$66 \quad 6$
Algiers.
From the same.

Otus vulgaris Fleming. France.

From the same.

" BRAchyotos (Gmel.) Labrador. From the same.

Syrnium aluco (Linn.) France. From the same.

" NEBUlosum (Gmel.) U. States. From the same.

Nyctale funerea (Linn.) France. From the same.

Buвo maximus Sibb. France.

From the same.

" virginianus Briss. Maryland. Presented by A. L. Heermann, M. D.

" ascalaphus Savigny, Africa. From Mons. Des Murs' Collection.

" Maculosus (Vieill.) Africa. From the same.

Ephialtes scops (Linn.) France. From the same.

" Asto (Linn.) Pennsylvania. Presented by S. W. Woodhouse, M. D.

Nyctea nivea (Daud.) Arctic America. From Mons. Des Murs' Collection.

Arhene noctua (Retzius.) France. From the same.

" Nana (Vigs.) S. America. From the same.

" sisu D’Orb. Cuba. From Mons. La Sagra's Collection.

"6 cucuroldes (Vig.) India. Presented by T. B. Wilson, M. D.

"t Brodiei (Burt.) India. Presented by Mr. E. Wilson.

" воовоок (Lath.) Australia. From Mr. J. Gould's Collection.

Suzva ulula (Linn.) Arctic America. From Mons. Des Murs' Collection.

\section{$2 d$ Order PASSERES.}

\section{Tribe FISSIROSTRES.}

\section{Family CAPRIMULGID $Æ$.}

Steatoris caripensis Humboldt. New Grenada. From Mons. Des Murs' Collection.
“6
66
S. America.
From the same.

Podargus Cuvieri Vig. and Horsf. Australia. From Mr. J. Gould's Collection.

"6 BRAchypterys Gould. Australia. From the same.

"6 Ilumeralis Vig. and Horsf. Australia. From the same.

Agotheles Nove Hollandix (Lath.) Australia. From the same.

Caprinulgus Europerus Linn. France. From Mons. Des Murs' Collection.
"6 AFrinis Horsf. Sumatra.
From the same.

Nyctibius Levcopterus (De Wied.) S. America. From the same.

Chordeiles virginianus (Briss.) Virginia and Pa. From the same.

Lurocalis leucopygius (Spix.) Paraguay. From the same.

Eurostopodus albogularis (Vig. and Horsf.) Australia. From Mr. J. Gould's Collection.

(: Gijtatus (Vig. and Horsf.) Australia. From the same.

Stenopsis hirundinaceus (Spix.) Paraguay. From. Mons. Des Murs' Collection.

Antrostowus voctrerts (Wilson.) N. Jersey. Presented by Mr. S. Ashmead. 
II. Family HIRUNDINIDE.

Hraundo rustica Linn. France. From M. Des Murs' Collection.

" RUFa Vieill. Pennsylvania. From the same.

"6 Frontalis Quoy and Gaim. Australia. From Mr. J. Gould's

Collection.

"6 Nigricans Vieill. Australia. From the same.

"6 Ariel (Gould.) Australia. From the same.

"Lunifrons Say. Rocky Mountains. Presented by A. L. Heermann, M. D.

"6 Bicolor Vieill. Maryland. From the same.

"6 albiventer Bodd. Paraguay. From M. Des Murs' Collection.

" crayoleuca Vieill. Chili. From the same.

Crpselus melba (Linn.) France. From the same.

" APUS (Linn.) France. From the same.

" Cayennensis (Gmel.) Paraguay. From the same.

Macropteryx Klecho (Raffl.) Java. From the same.

Coldocalia nidifica (Lath.) Java. From the same.

" Fuciphaga (Thunb.) Java. From the same.

Acanthylis pelasgia (Linn.) Pennsylvania. Presented by Professor Baird.

Atticora leucosternon Gould. Australia. From Mr. J. Gould's Collection.

Progne purpurea (Linn.) Pennsylvania. Presented by Professor Baird.

Cotyle riparia (Linn.) France. From M. Des Murs' Collection.

" "6 Pennsylvania. Presented by Wm. Gambel, M. D.

“ Serripennis (Aud.) Pennsylvania. Presented by Professor Baird.

Chelidon urbisa (Linn.) France. From M. Des Murs' Collection.

III. Family CORACID Æ.

Coracias garruma Linn. Hungary. From M. Des Murs' Collection.

Eurystonus pacificus (Lath.) Australia. From Mr. J. Gould's Collection.

V. Family ALCEDINIDÆ.

Dacelo gigas (Bodd.) Australia. From Mr. J. Gould's Collection.

Alcedo ispida Linn. France. From M. Des Murs' Collection.

" Berylina Vieill. Java. From the same.

Certle alcyon (Linn.) Maryland. Presented by A. L. Heermann, M. D.

Corythornis cristata (Linn.) Africa. From M. Des Murs' Collection.

Alcyone pulchra Gould. Australia. From Mr. J. Gould's Collection.

Halcyon sancta Vig. and Horsf. Australia. From the same.

"G MACLEAYII Jard. and Selby. Australia. From the same.

“ Cranovenrris (Vieill.) Java. From M. Des Murs' Collection.

\section{Family MEROPID Æ.}

Merops apiaster Linn. France. From M. Des Murs' Collection.

" ornatus Lath. Australia. From Mr. J. Gould's Collection. 


\section{Tribe TENUIROSTRES.}

\section{Family UPUPID E.}

Upupa epops Linn. France. From M. Des Murs' Collection.

II. Family PROMEROPIDE.

Nectarina famosa (Linn.) Africa. From M. Des Murs' Collection.

" minima (Sykes.) Africa. From the same.

Diceum hirundinaceum (Lath.) Australia. From Mr. J. Gould's Coilection.

Certhiola flaveota (Linn.) Guadeloupe. From M. Des Murs' Collection.

\section{Family TROCHILIDÆ.}

Phetornis superciliosus (Linn.) Brazil. From M. Des Murs' Collection.

Polytmus jugularis (Linn.) Martinique. Presented by T. B. Wilson, M. D.

“ Simplex (Less.) Brazil. From M. Des Murs' Collection.

* erevirostris (Less-) Brazil. From the same.

" Corallirostris (Bourc. and Muls.) Brazil. From the same.

Topaza pelta (Linn.) Brazil. From the same.

Calothorax Lucifer (Swains.) Brazil. From the same.

Mellisuga Alexandri (Bourc. and Muls.) California. Presented by A. L. Heermann, M. D.

"6 Minima (Swains.) Jamaica. From M. Des Murs' Collection.

" moschita (Linn.) Guiana. From the same:

" cristata (Linn.) Brazil. From the same.

“ Delalandi (Vieill.) Brazil. From the same.

" aмethystina (Gmel.) Brazil. From the same.

Hyzocharis cerrulea (Vieill.) N. Grenada. From the same.

". Sapphirina (Gmel.) Brazil. Presented by T. B. Wilson, M. D.

" cyanogenys (Pr. Max.) Brazil. From the same.

" prasina (Less.) Guiana. From M. Des Murs' Collection.

\section{Family MELIPHAGID压.}

Entomophila albigularis Gould. Australia. From Mr. J. Gould's Collection. "6 Rufigularis Gould. Australia. From the same.

Glyciphila albiprons Gould. Australia. From the same. "6 ocdLaris Gould. Australia. From the same. " Melanops (Lath.) Australia. From the same.

Acanthorynchus tenurostris (Lath.) Australia. From the same. "6 SUPErciliosus Gould. Australia. From the same.

Meliphaga phrygia (Lath.) Australia.

66 ornata Gould. Australia.

6 PLUmula Gould. Australia.

6. sovora Gould. Australia.

66 Flavigula Gould. Australia.

66 Nove Hollandie (Lath.) Australia.

" Australasiana (Shaw.) Australia.

¿6 Mysticalis Gould. Australia.

\&. zongirostris Gould. Australia.
From the same.

From the same.

From the same.

From the same.

From the same.

From the same.

From the same.

From the same.

From the same. 
Axthochera mellivora (Lath.) Australia. From Mr. J. Gould's Collection. "6 Lunulata Gould. Australia. From the same.

" Carunculata (Lath.) Australia. From the same.

" Lewrin Vigs. and Horsf. Australia. From M. Des Murs' Collection.

Phyllornis aurifrons Temm. India. Presented by Mr. E. Wilson.

Tropidorhynchus corniculatus (Lath.) Australia. Presented by T. B. Wilson, M. D.

" cyanotis (Lath.) Australia. From Mr. J. Gould's Collection.

Manorhina farrula (Lath.) Australia. From the same.

6 obscura Gould. Australia. From the same.

Melithreptus lunulatus (Shaw.) Australia. From Mons. Des Murs' Collection.

" Atricapillus (Lath.) Australia. From the same.

" virescens (Wagler.) Australia. From the same.

" chloropsis Gould. Australia. From the same.

\section{Family CERTHID无.}

Furnarius rufus (Gmel.) Paraguay. From M. Des Murs' Collection. Cinclodes nigrofumosus (D’Orb. and Lafr.) Chili. From the same.

Synallaxis humicola Kittl. Chili. From the same.

Annumbius acuticaudatus (Less.) Paraguay. From the same.

"6 RUber (Vieill.) Paraguay. From the same.

Anabates striatus Spix. Paraguay. From the same.

Picolatpes brunneicapillus (Lafr.) Mexico. Presented by A. L. Heermann, M. D.

Certhia familiaris Linn. France. From M. Des Murs' Collection.

" costa Bailly. Italy. Presented by Mr. E. Wilson.

"Americana Pr. Bonap. Vermont. Presented by T. B. Wilson, M. D.

Tichidroma muraria (Linn.) Switzerland. Presented by A. L. Heermann, M. D.

Chimacteris scandens Temm. Australia. From Mr. J. Gould's Collection.

"6 RUFA Gould. Australia. From the same.

Sitta Europea Linn. France. From M. Des Murs' Collection.

"6 Sxriaca Ehrenb. Dalmatia. From the same.

"6 Carolinensis Lath. Virginia. From the same.

Troglodytes parvulus Koch. France. From the same.

" "6 England. Presented by Mr. E. Wilson.

" hremalis Vieill. Canada. Presented by T. B. Wilson, M. D.

«DoN Vieill. Maryland. Presented by A. L. Heermann, M. D.

"6 Brevirostris Aud. Massachusetts. From Mons. Des Murs'

Collection.

" platensis (Lath.) Paraguay. From the same.

" Ludovicianus (Lath.) South Carolina. Presented by T. B. Wilson, M. D.

" arundinaceus Vieill. Pennsylvania. Presented by A. L. Heermann, M.D.

"BEwickil Aud. Maryland. From M. Des Murs' Collection.

"6 chrlensis Less. Brazil. From the same.

"Hortensis Less. Chili. From the same. 
Campromirxcus palurscexs (Lafr.) Australia. From Mr. J. Gould's Collection.

\section{Tribe DENTIROSTRES.}

\section{Family LUSCINID\&.}

Orthotomes sepicm Horsf. India. From M. Des Murs' Collection.

6 LoNgicaudes (Gmel.) India. From the same.

Primia faniliaris Horsf. India. Presented by Mr. E. Wilson.

Dryaorca cisticola (Temm.) Sicily. From M. Des Murs' Collection.

66 Tetrix (Vieill.) Africa. From the same.

" Maculosa (Bodd.) Africa. From the same.

"6 Ruficers (Gould.) Airica. From the same.

Calamanthus fuligivosus (Vig. and Horsf.) Australia. From Mr. J. Gould's Collection.

$\because$ Campestris Gould. Australia.

From the same.

Malurus craveus (Gmel.) Australia. From Mons. Des Murs' Collection.

$66 \quad$ India. Presented by Mr. E. Wilson.

“6 Loxgrcaudus Gould. Van Diemen's Land. From Mons. Des Murs' Collection.

" Elegans Gould. Australia. From Mr. J. Gould's Collection.

"Srlendens (Quoy and Gaim.) Australia. From the same.

"6 Melanocepiralus (Lath.) Australia. From the same.

“ Lainerti Vig. and Horsf. Australia. Presented by Mr. E. Wilson.

Stipiturus malachurus (Lath.) Australia. From Mr. J. Gould's Collection.

Amyts macroura Gould. Australia. From the same.

Cinclorhanphus cruralis (Vig. and Horsf.) Australia. From M. Des Murs' Collection.

" cantulans Gould. Australia. From Mr. J. Gould's Collection.

"6 strigatus (Lath.) Australia. From the same.

Megalurus rufescens (Vig. and Horsf.) Australia. From M. Des Murs' Collection.

" palustris Horsf. Java. From the same.

$\therefore$ Gramineus (Gould.) Australia. From Mr. J. Gould's Collection.

Calamodyta locustella (Penn.) France. From M. Des Murs' Collection.

"6 Melanopogon (Temm.) France. From the same,

66 phraguris (Bechst.) France. From the same.

66 CeTti (Marm.) France. From the same.

6 Aquatica (Lath.) Germany. From the same.

"L Luscinoides (Sav.) Piedmont. From the same,

"s arundinaces (Linn.) France. From the same.

"6 olrvetorua (Strickl.) Greece. From the same.

" Elatca (Linderm.) Greece. From the same.

" TAlustris (Bechst.) Switzerland. From the same.

" Lovgroostris (Gould,) Australia. From Mr. J. Gould's Collection.

66 Australis (Gould.) Australia. From the same.

66 afrins (Hodgs.) India. Presented by Mr. E. Wilson.

Juscinia Major Briss. France. From M. Des Murs' Collection.

$66 \quad 66 \quad$ Algiers. From the same.

66 pullonela Pr, Bonap. France. From the same. 
Edon galactodes (Temm.) Greece. From the same.

Sylvia melayoceriala (Gmel.) France. From the same. coxspicillata Marm. Sardinia. From the same.

"6 subatisisa Bonn. Dalmatia. From the same.

"T Trdata (Bodd.) Europe. Presented by 'T. B. Wilson, M. D.

"R Ruprelin Temm. Algiers. From M. Des Murs' Collection. curruca Lath. France. From the same.

atricapilla (Briss.) France. From the same. Crierea (Briss.) France. From the same. nortersis (Penn.) France. From the same. onpнea Temm. France. From the same. "6 Algiers. From the same. visoria Bechst. France. From the same. mirpolais (Linn.) France. From the same. "6 Tangier. From the same. Trochilus (Linn.) France. From the same. sibilatrix (Bechst.) France. From the same. rufa (Lath.) France. From the same. Boyellir (Vieill.) France. From the same.

Regtlus cristates Ray. France. From the same. "Ignicapildus Brehm. France. From the same.

Culicivora cerulea (Gimel.) Pennsylv. Presented by A. L. Heermann, M.D.

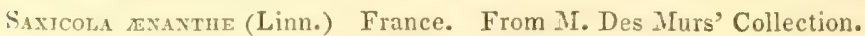

"s Stapazina (Linn.) France. From the same.

" Aurita Temm. France. From the same.

"6 Levconiela (Pall.) Dalmatia. From the same.

" Leucura (Gmel.) France. From the same.

"6 Tileata (Gmel.) Africa. From the same.

$\because$ Caprata (Linn.) Africa. From the same.

"6 explorator (Vieill.) Africa. From the same.

Pratixcola rubetra (Linn.) France. From the same. " Rubicola (Linn.) France. From the same. "6 " Africa. From the same.

Ruticilda pianicurus (Linn.) France. From the same. " тітичs (Scop.) France. From the same.

Erytuacus rubecula (Linn.) France. From the same. 66 "6 deformed egg. France. From the same. "6 " Africa. From the same.

Cyanecula suecica (Linn.) Germany. From the same.

Prtrojca fusca Gould. Australia: From the same.

"s Multicolor (Gmel.) Australia. From the same.

" cuculdata (Lath.) Australia. From Mr. J. Gould's Collection.

"G Goodenovir (Vig. and Horsf.) Australia. From the same.

"6 Tym nicea Gould. Australia. From the same.

“ Inodixogastra (Drapiez.) Australia. From Mons. Des Murs' Collection.

Sialia Wilsoni Swains. Pennsylvania. Presented by Wm. Gambel, M. D. " arctica Swains. N. W. Coast. Presented by T. B. Wilson, M. D.

Accextor alpixus (Gmel.) Switzerland. From M. Des Murs' Collection. "6 Modulafis (Linu.) France. From the same.

Enicocicital aurocapilius (Wils.) N. Jersey. Presented by A. L. Heermann, M.D.

"s Noveboraceisis (Gmel.) Massachusetts. Presented by T: B. Wilson, M. D. 
Serricorvis frontalts (Vig. and Horsf.) Australia. From MIr. J. Gould's

Collection.

" In $\quad$ minis Gould. Australia. From the same.

6: citreoguraris Gould. Australia. From the same.

"6 osculans Gould. Australia. From the same.

"Maculatus Gould. Australia. From the same.

Acantiriza pusilla (Lath.) Australia. From the same.

"6 ChrysorrhoA (Quoy and Gaim.) Australia. From the same.

"Dieneyensis Gould. Australia. From the same.

"6 Apicalis Gould. Australia. From the same.

is Ewivgir Gould. Australia. From the same.

" culicivora (Gould.) Java. From M. Des Murs' Collection.

"6 Fusca (Gould.) Australia. From Mr. J. Gould's Collection.

" brevirostris (Gould.) Australia. From the same.

Parus major Linn. France. From M. Des Murs' Collection.

"6 Careuleus Linn. France. From the same.

"ater Linn. France. From the same.

"6 Palustris Linn. France. From the same.

s6 Borealis Selys. Lougch. Furope. Presented by Mr. E. Wilson.

" Caudatus Linn. France. From M. Des Murs' Collection.

"6 atricapillus Linn. Pennsylvania. From the same.

“Carolinexsis Aud. Pennsylvania. Presented by A. L. Heermann, M. D.

"a ATRICeps Horsf. Java. From M. Des MIurs' Collection.

Lophophanes cristatus (Linn.) France. From the same.

"BIColor (Linn.) Pennsylvania. Presented by A. L. Heermann, M. D.

Psaltria mivia (Towns.) California. From the same.

Parotdes Pendulinus (Linn.) France. From M. Des Murs' Collection.

66 BLARMicus (Linn.) Germany. From the same.

Conirostrum ornatuar. California. Presented by A. L. Heermann, M. D.

Sprienostona leucopsis (Gould.) Australia. From Mr. J.Gould's Collection.

Miotilta varia (Lath.) N. York. Presented by T. B. Wilson, M. D.

Sycvicola festiva (Gmel.) Pennsylv. Presented by A. L. Heermann, M. D.

"6 Drscolor (Vieill.) N. Jersey. From M. Des Murs' Collection.

"s striata (Gmel.) Bay of Fundy. Presented by T. B. Wilson, M.D.

"B Backburnia (Gmel.) Massachusetts. From the same.

"6 americana (Linn.) Massachusetts. From the same.

" CHRYsottera (Linn.) Virginia. From the same.

66 Pennsyluanica (Linn.) Vermont. From the same.

"6 virens (Gmel.) Massachusetts. From M. Des Murs' Collection.

Helevata rubricapilla (Gmel.) Nova Scotia. Presented by T. B. Wilson, M. D.

"Swainsonir (Aud.) S. Carolina. From the same.

Trichas marilandicus (Briss.) Penn. Presented by A. L. Heermann, M. D.

Zosterors lateralis (Lath.) Van Diemen's Land. From Mons. Des Murs'

\section{Collection.}

"6 albogularis Gould. Australia. From Mr. J. Gould's Collection.

"chlorovotus Vieill. Australia. From the same.

Motacilla alba Linn. France. From M. Des Murs' Collection.

" Lugubris Temm. France. From the same.

"6 Boarula Penn. France. From the same.

"citreola Pall. France. From the same. 
Motacilla flava Linn. France.

From M. Des Murs' Collection.

" Yarrelli Gould. England. Presented by Mr. E. Wilson.

" Luzoniensis Scop. India. Presented by 'T. B. Wilson, MI. D.

" Capensis Linn. Africa. From M. Des Murs' Collection.

Exicures Lescievaulti (Tieill.) Java. From the same.

"6 Fuficapilus Temm. Java. From the same.

Grameina crenoleuca (Lath.) Australia. From Mr. J. Gould's Collection.

Antuls spivoletra (Linn.) France. From M. Des Murs' Collection.

"6 PRAtexsis (Linn.) France. From the same.

$"$ Arioneus Bechst. France. From the same.

$6 \quad 66 \quad$ Algiers. From the same.

" Campestris Bechst. France. From the same.

"Ludovicranus (Gmel.) Labrador. From the same.

"6 osscura (Gmel.) Island of Feroe. From the same.

"s Richardil (Vieill.) France. From the same.

"chil Vieill. Buenos Ayres. From the same.

" CAPEXSIS (Iinn.) Africa. From the same.

"Cervinus (Pall.) Tangier. From the same.

"Iurus (Ginel.) Paraguay. From the same.

" Australis Vig, and Horsf. Australia. From Mr. J. Gould's Collection.

"Thavigaster Swains. Africa. From MT. Des Murs' Collection.

\section{Family TURDID正.}

Macrovus Cafistratus (Temm.) Java. From M. Des Murs' Collection.

"6 Melanothorax (Temm.) Java. From the same.

"s PrRuogenys (Temm.) Java. From the same.

Formicrvora caxtator (Bodd.) Africa. From the same.

Prtta cyayura (Gmel.) Java. From the same.

$\therefore$ strepitaxs Temm. Australia. From Mr. J. Gould's Collection.

Mrropmoves craveus (Horsf.) Java. From M. Des Murs' Collection.

Civclus aquaticus Bechst. France. From the same.

Turnus viscivorus Linn. France. From the same,

"6 Pilaris Linn. Germany. From the same.

" arusicus Linn. France. From the same.

"L ILIACUS Linn. France. From the same.

"s renula Linn. France. From the same.

"6 66 Tangier. From the same.

" Torouatus Linn. Switzerland. From the same.

" Cranes Linn. France. From the same.

"Saxatros Linn. Switzerland. From the same.

" atstelinus Gmel. Pennsylvania. From the same.

"6 Solitarius Wils. Massachusetts. From the same.

"s migratorius Linn. Pennsylvania. From the same.

"Naxus Aud. Nova Scotia. Presented by T. B. Wilson, M. D.

"Maglanicus King. Chili. From M. Des Murs' Collection.

"c crocur Vieill. Brazil. From the same.

" crotopezus Ill. Rio Janeiro. From the same.

"6 Carbonarius Ill. Rio Janeiro. From the same.

¿ Tubres Temm. Cubd. From M. La Sagra's Collection.

" Flavipes Vieill. Rio Janeiro. From M. Des Mus' Collectior.

"6 Crinereus Gmel. Buenos Ayres. From the same.

"6 Fuscatus Pall. Bahia. From the same.

"c citrinus Lath. India. Presented by Mr. E. Wilson.

" MCropus Hodgs. India. From the same,

"r Tolocermalus Lath. Australia. From Mr. J. Gould's Collection. 
Minus polyglottus (Linn.) Maryland. From M. Des Miurs' Collection.

" Carolinessis (Linn.) Pennsylvania. From the same.

"trienka (Mol.) Buenos Ayres. From the same.

"G Calandria (D'Orb. and Lafr.) New Grenada. From the same.

" " " Paraguay. From the same.

"sors.tis (D’Orb. and Lafr.) Chili. From the same.

Toxostonus rufus (Linn.) Pennsylvania. From the same.

Crxclosome punctatum (Lath.) Australia. From Mr. J. Gould's Collection.

"Castanotua Gould. Australia. From the same.

'Trualia pileatr Horsf. Java. From MT. Des Murs' Collection.

Pomatormines superciminers (Bl.) Australia. From Mr. J. Gould's Collection.

"TEmporalis Vig. and Horsf. Australia. From the same.

Íteria viridis (Gmel.) Pennsylvania. Presented by S. W. Woodhouse, MI.D.

Orioles gatbetus Linn. France. From Mr. Des Murs' Collection.

" CHNExsis Linn. Java. From the same.

Andropades mportunus (Vieill.) Airica. From the same.

Pycmonotus aurigastar (Vieill.) Africa. From the same,

" CApensis (Gmel.) Africa. From the same.

" crocorrhous Strickl. Java. From the same.

"Golavier (Scop.) Java. From the same.

"Jocosus (Linn.) India. Presented by Mr. E. Wilson.

"Leucogenys (Gray.) India. From the same.

III. Family MUSCICAPID Fi:

Lipaxgus reumbeus (Licht.) Rio Janeiro. From M. Des Murs' Collection.

textoptera nevgeta (Linn.) Buenos Ayres. From the same.

"Variegata (D'Orb. and Lafr.) Buenos Ayres. From the same.

Fluvicola climazura (Vieill.) Brazil. From the same.

" PICA (Bodd.) Paraguay. From the same.

" Nigerrima D'Orb. and Lafr. Rio Janeiro. From the same.

Lichenors perspicildati (Gmel.) Buenos Ayres. From the same.

Corurus filicarida (Spix.) Rio Janeiro. From the same.

Machetornis rixosa (Vieill.) Paraguay. From the same.

Scapiorhymchus pitangua (Linn.) Brazil. From the same.

Saurophagus sulpirtratus (Linn.) Brazil. From the same.

Tyranus intremidus Vieill. Pennsylvania. From the same.

" " $"$ Brazil. From the same.

« vertichiss (Say.) California. Presented by A. L. Heermann, M. D.

" Magnirostris D'Orb. Cuba. From M. La Sagra's Collection.

" Jemancholicus Vieill. Buenos Ayres. From MI. Des Murs' Collection.

" rusincts (Licht.) Paraguay. From the same.

Mrevelus typannus (Linn.) Buenos Ayres. From the same.

Tyraxinua crinita (Linn.) Pennsylvania. From the same.
66$$
66
$$
New Jersey. Presented by Wm. Gambel, M. D.
" audax (Gmel.) Buenos Ayres. From M. Des Murs' Collection.
" FErox (Gmel.) Brazil. From the same.
"Cooperir (Nutt.) Vermont. Presented by T. B. Wilson, M.D. 
Trranxula nuxciola (Wils.) Penn. Presented by S. W. Woodhouse, M. D.

" pricbe (Lath.) N. Hampshire. Presented by T. B. Wilson, M. D.

"a acadica (Gmel.) Pennsylvania. From Mr. Des Murs' Collection.

"Trailiti (Aud.) Vermont. Presented by T. B. Wilson, M.D.

" virexs (Linn.) Pennsylvanin. Presented by Professor Baird.

" "6 Cuba. From M. La Sagra's Collection.

"Thaviventris' Baird. Vermont. Presented by T. B. Wilson, M. D.

$"$ Nigricans Swains. California. Presented by A. L. Heermann, M.D.

"6 Cayenvensis (Linn.) Brazil. From M. Des Murs' Collection.

" albicolurs (Vieill.) Brazil. From the same,

Erania pagava (Licht.) Brazil. From the same.

" ICTerophrys (Vieill.) Paraguay. From the same.

Eescarthuls parulus (Kittl.) Chili. From the same.

Tityma Cuvieri (Swains.) Rio Janeiro. From the same.

Mescrvora rupestris (Pr. Max.) Rio Janeiro. From the same.

Ruipidura albiscapa Gould. Australia. From Mr. J. Gould's Collection. " hotacillomes Vig. and Horsf. Australia. From the same.

Tomitrea torquata (Gmel.) Africa. From M. Des Murs' Collection.

Monarcifa nigra (Sparr.) Brazil. From the same.

Seistra inquieta (Lath.) Australia. From Mr. J. Gould's Collection.

" NiTida (Gould.) Australia. From the same.

Hemichelidon latirostris (Rafll.) Java. From the same.

Muscicapa grisola Linn. England. Presented by Mr. E. Wilson.

" albicollis Temm. Germany. From M. Des Murs' Collection.

"G atricapilla Linn. France. From the same.

" Palka Bechst. Saxony. From the same.

Seropinga mitrata (Lath.) Missouri. From the same.

" Ruticilla (Gmel.) Pennsylvania. Presented by Mr. S. Ashmead.

Vireo noveboracensis (Gmel.) Massachusetts. Presented by T. B. Wilson, M. D.

66 solitarius (Wils.) N. York. From the same.

"6 Flavifrons (Gmel.) Massachusetts. From M. Des Murs' Collection.

"6 olivaceus (Linn.) N. Jersey. Presented by Mr. S. Ashmead.

"Gil.vus (Vieill.) Pennsylvania. Presented by Professor Baird.

\section{Family AMPELIDÆ.}

Pardalotes runctatus (Lath.) Van Diemen's Land. From Mr. J. Gould's Collection.

" Striatus (Lath.) Van Diemen's Land. From the same.

" Quadragintus Gould. Van Diemen's Land. From the same.

" BREvirostris (Gould.) Australia. From the same.

Pacirycephala gutturalis (Lath.) Australia. From the same.

" Ivfriventris (Lath.) Australia. From the same.

"Inornata Gould. Australia. From the same.

Eopsaltria australis (Lath.) Australia. From the same.

" Griseogularis Gould. Australia. From the same.

Pipra caudata Shaw. Bahia. From M. Des Murs' Collection.

" Militaris Shaw. Bahia. From the same.

" rubrocapilla Briss. Bahia. From the same.

". Leucocrlea Linn. Bahia. From the same. 
Rupicola peruviana (Lath.) New Grenada. From the same.

Ampels cedrorum (Vieill.) Pennsylvania. From the same.

Procxias variegata (Linn.) Brazil. From the same.

Campepiaga Macei (Less.) India. Presented by T. B. Wilson, M.D.

"6 mela vops (Lath.) Australia. From Mr. J. Gould's Collection.

"6 T'Arvirostris Gould. Australia. From the same.

"6 Mentalis (Vig. and Horsf.) Australia. From the same.

" Humeralis (Gould.) Australia. From the same.

Artauts crnereus Vieill. Australia. From the same.

" sordidus (Lath.) Australia. From the same.

" teucopygialis Gould. Australia. From the same.

"6 Tersonatus Gould. Australia. From the same.

"superciliosus Gould. Australia. From the same.

Dicrurus balicassius (Linn.) Java. From M. Des Murs' Collection.

" Macrocericus Vieill. Java. From the same.

\section{Family LANIID无.}

Lanius excubrtor Linn. France. From M. Des Murs: Collection.

6. Merintonalis 'Temm. France. From the same.

"6 Ludovicianus I.inn. S. Carolina. Presented by T. B. Wilson, M. D.

"sirnor Gmel. France. From M. Des Murs' Collection.

"Collaris Linn. Africa. From the same.

" Borealis Vieill. Labrador. Presented by Mr. E. Wilson.

Enneoctonus collurio (Linn.) France. From M. Des Murs' Collection. " Rufus (Briss.) France. From the same.

Telophorus zerlonus (Linn.) Africa. From the same.

Oreoica cristata (Lewin.) Australia. From Mr. J. Gould's Collection.

Coljuriocincla marmonica (Lath.) Australia. From the same.

" Brunnea Gould. Australia. From the same.

" RUfiventris Gould. Australia. From the same.

" parvula Gould. Australia. From the same.

"6 Seleri Gould. Australia. From Mr. Des Murs' Collection.

Lantarius boulboul (Lath.) Africa. From the same.

Cracticus cinereus (Gould.) Australia. From the same.

" Torquatus (Lath.) Australia. From Mr. J. Gould's Collection.

"6 Nigrogularis (Gould.) Australia. From the same.

" Levcopterus Gould. Australia. From the same.

\section{Tribe CONIROSTRES.}

I. Family CORVID无.

Gymormina tibicen (Lath.) Australia. From Mr. J. Gould's Collection. "6 ORganicum Gould. Australia. From the same.

" anaphonensis (Temm.) Australia. From the same.

Strepera graculina (Shaw.) Australia. From M. Des Murs' Collection.

"6 Arguta Gould. Australia. From Mr. J. Gould's Collection.

Garrulus glandarius (Linn.) France. From M. Des Murs? Collection.

"6 atricapillus Geoffr. Spain. From the same.

Cyavocorax cristatus (Linn.) Virginia. From the same.

"Steli.eri (Gmel.) California. Presented by T. B. Wilson, M. D. 


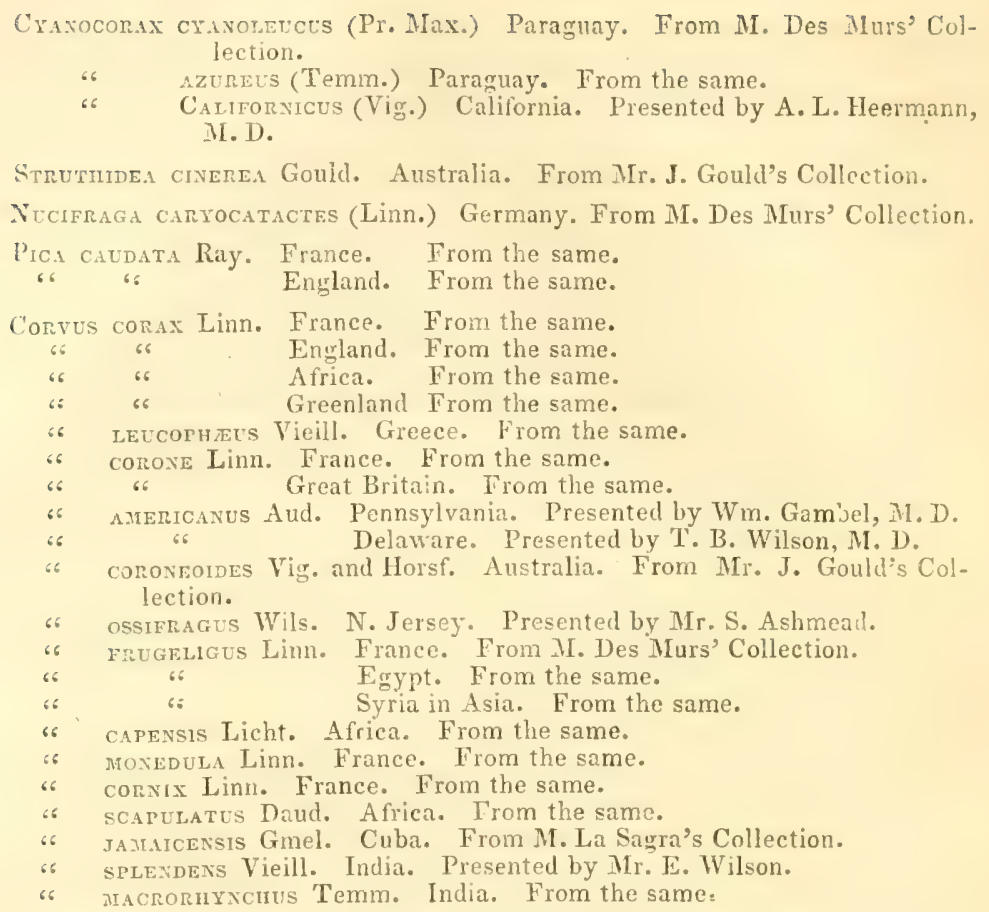

Pyrmiocorax melanoruxycius (Vieill.) Australia. From Mr. J. Gould's Collection.

Corcorax alpinus Vieill. Switzerland. Presented by Mr. E. Wilson. Coracha gracula (Linn.) Italy. From M. Des Murs' Collection.

\section{Family STURNIDÆ.}

Astrapia caruxculata (Less.) Africa. From M. Des Murs' Collection. Juida nabouroup (Daud.) Africa. From the same. Gracula religiosa Linn. Sumatra, From the same.

66 India. Presented by Mr. E. Wilson.

Acridotheres Tristis (Linn.) Africa. From M. Des Murs' Collection. Hetarornis cristatella (Linn.) Java. From the same.

Sturnopastor jalla Horsf. Java. From the same.

Sturnus vulgaris Linn. France. From the same.

66 Uxicolor Marm. Italy. From the same.

66 Africa. From the same.

Stursela Ludovicianus (Linn.) N. Iork. From the same.
$66 \quad 66$
Penna. Presented by Wm. Gambel, M. D.
66 Neglaecta Aud. California. Presented by A. L. Heermann, M. D.
" inprocreris Wagler. Cuba. From M. La Sagra's Collection.
" Milraris (Linn.) Chili. From M. Des Murs' Collection. 
Scolecopiragus ferrugineus (Wils.) Nova Scotia. Presented by T. B, Wilson, M.D.

Quiscalus purrureus (Licht.) Maryland. Presented by A. L. Hermann, M. D.

"6 Major Vieill. S. Carolina. From M. Des Murs' Collection.

$\because$ porruratus Swains. Mexico. From the same.

" barita Gmel. Brazil. From the same.

" "6 Cuba. From M. La Sagra's Collection.

Scapindurus atroviolaceus D'Orb. Cuba. From the same.

Cacicus persicus (Linn.) Para. Presented by MIr. W. H. Edwards.

" HeMorrious (Linn.) Para. From the same.

" solitarius (Vieill.) Paraguay. From M. Des Murs' Collection.

ICteres velgaris Daud. Paraguay. From the same.

" " Buenos Ayres. From the same.

"Jamaicait (Gmel.) Rio Janeiro. From the same.

Xantioryus varius (Gmel.) Pennsylvania. From the same.

"6 Dosinicensis (Linn.) Cuba. From M. La Sagra's Collection.

$\because \quad$ Aurantius (Valenc.) Paraguay. From M. Des Murs' Collection.

"Glavus Daud. Buenos Ayres. From the same.

$\because$ virids (Gmel.) Buenos Ayres. From the same.

Yrandres Balmimore (Linn.) Pennsylvania. Presented by Mr. S. Ashmead.

"B-Bullocki Swains. California. Presented by A. L. Heermann, M.D.

Molothrus Pecoris (Gmel.) Pennsylvania. Presented by Wm. Gambel, M.D.

" Bonariexsis (Gmel.) Chili. From M. Des Murs' Collection.

Agecaius raeniceus (Linn.) N. Jersey. From the same.

"6 TRicolor Aud. California. Presented by A. L. Heermann, MI, D.

"6 Gubernator (Wagl.) California. From the same.

" Tanthocerilalus (Pr. Bonap.) California. From the same.

“ CRrfates (Mol.) Chili. From M. Des Murs' Collection.

$\therefore$ pyrrhorterus (Vieill.) Brazil. From the same.

"6 aterrimus (Kittl.) Chili. From the same,

" Brevirostris (D'Orb. and Lafr.) Paraguay. From the samc.

"G Tillius (Mol.) Chili. From the same.

Chrysonus frontalts (Vieill.) Buenos Ayres. From the same.

Dolichonyx onyzivorus (Linn.) Massachusetts. From the same.

IV. Family FRINGILLID A.

Hyphantornis Capensis (A. Smith.) Africa. From M. Des. Murs' Collection. "6 aurantia (Vieill.) Africa. From the same.

Ploceus manyar (Horsf.) India. Presented by Mr. E. Wilson.

"6 Nelicovrvi (Scop.) Bengal. From Mí. Des Murs' Collection.

"6 Aureus (Gmel.) Africa. From the same.

" Oryx (Linn.) Africa. From the same.

" MaDAGASCARIENsis (Linn.) Island of Bourbon. From the same.

"Jonquillaceus Vieill. Java. From the same.

Gurraca cerrulea (Linn.) Pennsylvania. Presented by T. B. Wilson, M.

" iudoviciana (Linn.) Vermont. From the same.

Calanospiza bicolor (Towns.) Missouri. Presented by A. L. Heemann, M. D.

Cardinalis virginianus Pr. Bonap. Maryland. From the same.

"6 Carlsonir (Daud.) Brazil. From M. Des Murs' Collection.

"6 Laid at Paris. From the same. 
Coccotminatstes vulgaris Briss. France. From the same.

Pirieo erythroptinlia (Linn.) N. Jersey. From the same.

is Arctica Swains. California. Presented by A. L. Heermann, M. D.

" Fusca Swains. California. From the same.

P'ityidus arelanocephalus (Swains.) California. From the same.

Salatator cerulescens Vieill. Buenos Ayres. From Mr. Des Murs' Collection.

"6 aurantiarostris Vieill. Buenos Ayres. From the same.

Rifaimphoris brasilia (Linn.) Para. Presented by T. B. Wilson, M. D.

" Jacapa (Linn.) Bahia. From M. Des IIurs' Collection.

Prrayga rugra (Linn.) Pennsylvania. Presented by Mr. S. Ashmead.

“ שstiva (Gmel.) Brazil. From M. Des Murs' Collection.

Tixigra emiscopts Linn. Bahia. From the same.

is ornata Sparr. Paraguay. From the same.

" zeva (Linn.) Brazil. From the same.

" OLIVAScens Licht. Rio Janeiro. From the same.

Thamphonts letcomerts (Gmel.) Paraguay. From the same.

"6 Cristatus (Linn.) Brazil. From the same.

Calliste festiva (Shaw.) Brazil. From the same.

"6 thoracica ('Temm.) Rio Janeiro. From the same.

" Braziliensis (Linn.) Bahia. From the same.

" Cayanna (Limn.) Brazil. From the same.

Etrionra violacea (Linn.) Bahia. From the same.

Euplectes carensis (Linn.) Africa. From the same.

Muxia oryzivora (Linn.) Java. From the same.

6 princtularia (Linn.) Java. From the same.

" rasa (Linn.) Java. From the same.

" striata (Linn.) Java. From the same.

" Cantans (Gmel.) Africa. From the same.

“ Malacca Linn. Africa. From the same.

Amadina fasciata (Gmel.) Africa. From the same.

P'ennila cincta (Gould.) Australia. From Mr. J. Gould's Collection.

Sporotmlastes castanotis (Gould.) Australia. From the same.

" TEMPoralis (Lath.) Australia. From the same.

6 Nitida (Lath.) Australia. From the same.

"Guttata (Shaw.) Australia. From the same.

Stegayorleura modesta (Gould.) Australia. From the same.

"6 Ruficauda (Gould.) Australia. From the same.

:G Bichexovil (Vigors.) Australia. From the same.

Fistrelda artandava (Linn.) Africa. From M. Des Murs' Collection.

" Astrild (Linn.) Africa. From the same.

Plectropitanes nivalis (Linn.) Iceland. From the same.

"6 Lapponica (Linn.) Greenland. From the same.

" orvatus (Towns.) Rocky IIts. Presented by A. L. Heermann, M. D.

Cynchramus miliaria (Linn.) France. From M. Des Murs' Collection.

Scuzxicola schexiculus (Linn.) France. From the same.

Emberiza oitrineld Linn. France. From the same.

" " 6 Africa. From the same.

"6 Hortulana Linn. France. From the same.

" cIRLA Linn. France: From the same. 
Eyberiza cia Linn. France. From M. Des Murs' Collection.

"6 Druca (Molina.) Chili. From the same.

Fringlularia flaviventris (Vieill.) Africa. From the same.

Euspiza Melanoceritala (Scop.) Greece. From the same.

"6 Americana (Gmel.) Pennsylva. Presented by T. B. Wilson, M. D.

Gubernatrix cristatelua (Vieill.) Buenos Ayres. From M. Des Murs Collection.

Paroaria cuculiata (Lath.) S. America. From the same.

Volatinia splemens (Vieill.) S. America. From the sime.

" Jacarina (Linn.) S. America. From the same.

Sriza ciris (Linn.) S. Carolina. From the same.

" CYanea (Linn.) Pennsylvania. Presented by Professor Baird.

"AMEva (Say.) California. Presented by A. L. Heermann, M. D.

Strutuus myemalis (Linn.) Alleghany Mts. Presented by Mr. S. Ashmead.

Pirzygilus alaudina (Kittl.) Brazil. From M. Des Murs' Collection.

Zonotricira amicolirs (Gmel.) Halifax. Presented by T. B. Wilson, M. D.

" Metodia (Wils.) Pennsylvania. From M. Des Murs' Collection.

"Graminea (Gmel.) Pennsylvania. From the same.

"Atricipilla (Aud.) California. Presented by A. L. Heermann, M. 1 .

" matutina (Licht.) Rio Janeiro. From M. Des Murs' Collection.

Crondestes gramiaca (Say.) Missouri. Presented by T. B. Wilson, M. D.

Spizelya siattucisil (Aud.) Missouri. From the same.

" Canadensis (Lath.) Nova Scotia. From the same.

" soctalis (Wils.) Pennsylvania. Presented by Wm. Gambel, M. D.

"6 posilia (Wils.) Pennsylvania. From M. Des MIurs' Collection.

Passerculus palustris (Wils.) Vermont. Presented by T. B. Wilson, M. D.

Peucra Bacimanit (Aud.) S. Carolina. Presented by T. B. Wilson, M.D.

Coturniculus Hrastowir (Aud.) N. Jersey. From the same.

“ Passeriva (Wils.) Penna. From M. Des Murs' Collection.

"6 manime (Licht.) Brazil. From the same.

Amodramus maritimus (Wils.) N. Jersey. Presented by A. L. Heermann, M.D.

“ Caudacutus (Gmel.) N. Jersey. From M. Des Mrurs' Collection.

Phonipara lepidus (Linn.) Cuba. From M. La Sagra's Collection.

Sperzophila mysia (Vieill.) Bahia. From M. Des Murs' Collection.

Fringilla calebs Linn. France. From the same.

Passer montana (Linn.) France. From the same.

$"$ Dourstica (Linn.) France. From the same.

6 66 Africa. From the same.

"Italis (Vieill.) Greece. From the same.

"Salicicola?(Vieill.) Spain. From the same.

"Arcuata (Gmel.) Africa. From the same.

Petronia stulta (Gmel.) France. From the same.

Cinorostiza chloris (Linn.) France. From the same.

Chrysonitris tristis (Linn.) Pennsylvania. Presented by Professor Baird.

"Lawrexcir (Cassin.) California. Presented by A. L. Heermann, M.D.

Carysonitris punus (Wils.) Labrador. Presented by T. B. Wilson, MI. D. 
Carduelis elegans Steph. France. From M. Des Murs' Collection.

Alario auraytia (Gmelo) Africa. From the same.

Citrinfela vigriceps (Rupp.) Africa. From the same.

Crithagea brasiliexsis (Gmel.) Brazil. From the same.

" Luteocerilala (D'Orb.) Bahia. From the same.

" Flaveola (Linn.) Paraguay. From the same.

Buserives sulpiuratus (Linn.) Africa. From the same.

Serinus butraceus (Linn.) Africa. From the same.

" Canaria (Linn.) Canary Islands. From the same.

"GERIDIovalis Bonap. France. From the same.

I'yrriela coccives (Sandb.) Europe. Presented by Mr. E. Wilson.

"6 Rubicilla Pall. France. From M. Des Murs' Collection.

Loxia pityopsittacus Bechst. Germany. From the same.

" curvirostra Linn. Germany. From the same.

"Auericana Wils. Vermont. Presented by T. B. Wilson, M. D.

Carrodacus pamimaris M'Call. California. Presented by A. L. Heermann, M. D.

" purpureus (Gmel.) Mass. From M. Des Murs' Collection.

" Frontalis (Say.) California. Presented by Col. M'Call.

Montrmingilla nivalis (Linn.) Switzer'and. From M. Des Murs' Collection.

Linota caxidina (Linn.) France. From the same.

"Moxtusi (Gmel.) France. From the same.

" " Europe. Presented by T. B. Wilson, M. D.

Acantiris linaria (Linn.) Scotland. From the same.

" " $\quad$ Labrador. From M. Des Murs' Collection.

" Borealis (Temm.) Greenland. From the same.

Alauda arvensis Linn. France. From the same.

" brachydaryla Temm. France. From the same.

" cristata Linn. France. From the same.

" ARBOREA Linn. France. From the same.

"c crassirostris (Vieill.) Africa. From the same.

" Ruficeps Rupp. Africa. From the same.

Melanocoryma calandra (Linn.) France. From the same.

Otocoris ALpestris (Linn.) Labrador. From the same.

". rufa (Aud.) California. Presented by Wm. Gambel, M.D.

Megalopionus rufipileus (Vieill.) Africa. From M. Des Mlurs' Collection.

Phyrotoma rara Moll. Chili. From the same.

V. Family COLID $\mathbb{E}$.

Colius striatus Gmel. Africa. From M. Des Murs' Collection.

VI. Family MUSOPHAGID 2 .

Turacus erytirolophus (Vieill.) Africa. From M. Des Murs' Collection.

Oristrocomus cristatus (Lath.) Paraguay. From the same. 


\section{$3 d$ Order SCANSORES.}

II. Family PSITTACID E.

Nrammous Nove Holdanie (Gmel.) Australia. From Mr. J. Gould's Collection.

Peatrcerces Brownil (Kuhl.) Australia. From the same.

" SFimorquatus Quoy and Gaim. Australia. From the same.

"6 ICTERotis (Temm.) Australia. From the same.

" pileatus Vig. Australia. From the same.

"6 Eximus (Shaw.) Australia. From the same.

"Barmardir (Lath.) Australia. From the same.

"zoxarius (Shaw.) Australia. From the same.

P'sempotus nematonotus Gould. Australia. From Mr. J. Gould's Collection.

Aprosmictus scapulatus (Bechst.) Australia. From the same.

Polvtelis melanura (Vig.) Australia. From the same.

Paleoris rufirostris (Linn.) Africa. From M. Des Murs' Collection.

"Alexandri (Linn.) Asia. From the same.

"6 Cubicularius (Linn.) Asia. From the same.

"Bengalensis Briss. India. Presented by Mr. E. Wilson.

Mlelorsittacus undulatus Shaw. Australia. From Mr.J. Gould's Collection.

Lathanus Nove Zealandie (Sparr.) New Zealand. Presented by 'T. B. Wilson, M. D.

Fuphema elegays Gould. Australia. From Mr. J. Gould's Collection.

"Aurantra Gould. Australia. From the same.

"6 chrysostona (Kuhl.) Australia. From the same.

¿6 Pulcielda (Shaw.) Australia. From the same.

Trichorlossus porphyoecepiralus Diet. Australia. From the same.

"6 pusillus (Shaw.) Australia. From the same.

"6 concinnus (Shaw.) Australia. From the same.

Ara ararauna (Linn.) Brazil. From M. Des Murs' Collection.

Conurus jexuara (Gmel.) From the same.

"6 Movacius (Bodd.) From the same.

Eos ruma (Gmel.) Molucca. From the same.

Eclectus Cerlonensis (Bodd.) E. Indies. From the same.

Psittacus errtracus Linn. Senegal. From the same.

Curysotis estruus (Linn.) Brazil. From the same.

"6 Festuves (Linn.) Brazil. From the same.

66 ochrocephatus (Gmel.) Brazil. From the same.

$\because$ Amazonicus (Briss.) Brazil. From the same.

Pullaria guineensis (Briss.) Africa. From the same.

Cacatua sulpuurea (Gmel.) N. S. Wales. From Mr. J. Gould's Collection. "6 Moluccensis (Gmel.) Molucea Isl. From M. Des Murs' Collection. "6 Cristata (Linn.) Molucca Isl. From the same.

Licmetis pastinator Gould. Australia. From Mr. J. Gould's Collection.

66 Tevurostris (Kuhl.) Australia. From the same.

Carpptoruxnchus maso Gould. Australia. From the same. 66 Baudinil Lear. Australia. From the same. 
III. Family PICID无.

I armodox marothorax (Cuv.) Africa. From M. Des Murs' Collection. " Levcomelas (Bodd.) Africa. From the same.

l'rcus major Linn. France. From the same.

"s Menus Linn. France. From the same.

" MiNor Linn. France. From the same.

" LEUCoxotus Bechst. Germany. From the same.

“ PUbescens Linn. Maryland. Presented by A. L. Heermann, M. D.

" Martive Aud. Vermont. Presented by T. B. Wilson, MI.D.

" analis Temm. Java. From M. Des Murs' Collection.

Drrcorrs martius (Linn.) Europe. Presented by Mr. E. Wilson.

Dexprobates Percussus (Temm.) Cuba. From M. La Sagra's Collection.

Gecixes vininis (Linn.) France. From M. Des Murs' Collection.

"Cands (Gmel.) Germany. From the same.

Mefaverres eryruroceprialus (Linn.) Pennsylvania. Presented by Wm. Gambei, M. D.

Colartes auratus (Limn.) Pennsylvania. From the same.

"Superciliosus Vig. Cuba. From MI. La Sagra's Collection.

Yuxx torqulut Linn. France. From Mi. Des Murs' Collection.

IV. Family CUCULID E.

Saurothera Merhini D'Orb. Cuba. From M. La Sagra's Collection.

Cextrorus Affinis Horsf. Java. From M. Des Mirss' Collection. "6 Timasianus (Lath.) Australia. From Mr. J. Gould's Collection.

Dirlofterts gutra (Gmel.) Buenos Ayres. From Mr. Des Murs' Collection. " Chocin (Vieill.) Paraguay. From the same.

Praya cayana (Linn.) Brazil. From the same.

Coccrzus americanus (Linn.) Pennsylvania. Presented by Professor Baird.

" Erytiroptualius (Wils.) Maryland. Presented by A. L. Heermann, M.D.

Crotophaga ani Linn. Paraguay. From M. Des Murs' Collection. "6 "Cuba. From M. La Sagra's Collection.

"6 Major Linn. Brazil. From M. Des Murs' Collection.

" " $"$ Para. Presented by Mr. Wm. H. Edwards.

*6 Sulcirostra Swains. Peru. From M. Des Murs' Collection.

Scrtrrops Nove Holraxdie Lath. Australia. From Mr. J. Gould's Collection.

Cuculus caxorus Linn. France. From Mr. Des Mfurs' Collection.

" Inorvatus Vigs. and Horsf. Australia. From Mr. J. Gould's Collection.

" cinerascens Vig. and Horsf. Australia. From the same.

Ciralcites lucidus (Gmel.) Australia. From the same.

" osculus Gould. Australia. From the same.

\section{4 th Order COIUMBE. \\ I. Family COLUMBID $E$.}

Priloxopus puccierninus (Scop.) Java. From M. Des MIurs' Collection.

Tréron aromatxa (Gmel.) Jaya. From the same.

" verians (Linn.) Java. From the same. 
Carpopiraga myristicuvora (Scop.) Australia. From M. Des Murs' Collection. " Luctuosa (T'emm.) Australia. From Mr. J. Gould's Collection.

"6 Norfolciexsis (Lath.) Australia. From the same.

" Magnifica (Temm.) Australia. Presented by Mr. E. Wilson.

Cozuniba aenas Linn. Fngland. From M. Des Murs' Collection. Palumbus Linn. France. From the same. Livia Briss. France. From the same.

66 England. Presented by MIr. E. Wilson.

guinea Linn. Africa. From M. Des Murs' Collection.

araucana Less. Chili. From the same.

cerrlea Temm. Brazil. From the same.

Fitzroy King. Chili. From the same.

gyinoptiataios Temm. Brazil. From the same.

corensis Gmel. Brazil. From the same.

rufina Temm. Cayenne. From the same.

Ectopistes mitatortes (Linn.) Ohio. Presented by Wm. Gambel, M. D.

" Carolinensis (Linu.) Pennsylvania. From the same.

"6 66 Cuba. From M. La Sagra's Collection.

6. 66 California. Presented by A. L. Heermann, if. D.

Geopelia rumeratis (Temm.) Australia. From Mr. J. Goull's Collection.

" cuneata (Lath.) Australia. From the same.

"6 Traneulla Gould. Australia. From the same.

" striata (Linn.) Java. From MI. Des MTurs' Collection.

Macropygia amboinensis (Linn.) Java. From the same.

AEna Capexsis (Linn.) Island of Lucon. From the same.

Turtur auritus Ray. France. From the same.

"6 Birorquatus (T'emm.) Java. From the same.

6 66 Africa. From the same.

"6 Risorius (Linn.) France. Froin the same.

65 Chinensis (Scop.) Java. From the same.

Columbina picur (Temm.) Chili. From the same.

"Strepitans Spix. Brazil. From the same.

"Campestris Spix. Brazil. From the same.

Zexaida amabilis Pr. Bonap. Brazil. From the same.

66 AURITA (Temm.) Chili. From the same.

Cuamerelia passerixa (Linn.) Georgia. Presented by T. B. Wilson, M. D. "6 Talpacoli (Temm.) Brazil. From M. Des Murs? Collection.

"andisa (Linn.) Brazil. From the same.

Peristera montana (Linn.) Florida. Presented by T. B. Wilson, M. D.

Ocrpiats Lophotes (Temm.) Australia. From Mr. J. Gould's Collection.

Phars chalcoptera (Lath.) Australia. From the same.

"6 IIstrionica Gould. Australia. From the same.

"s ELEgans (Temm.) Australia. Presented by Mons. J. Verreaux.

Geophars Smithi (Jard. and Selby.) Australia. From Mr. J. Gould's Collection.

"s Scripta (Temm.) Australia. From the same.

Cilexas luzonica (Scop.) Java. Presented by T. B. Wilson, M. D. 


\section{5 th Order GALIIN E. \\ I. Family CRACID $\approx$.}

Ortajida ratraci (Bodd.) Brazil. From M. Des Murs' Collection.

Penelote riplle (Jacq.) Paraguay. From the same:

"6 cristata (Linn.) Mexico. From the same.

"6 Marail, Gmel. Brazil. From the same.

" obscura Illig. Brazil. From the same.

" pileata Licht. Paraguay. From the same.

" aburri Goudot. New Grenada. From the same.

Crax glodicera Linn. S. America. From the same.

Pauxi galleata (Lath.) Mexico. From the same.

\section{Family MEGAPODID $Æ$.}

Taldegaldus Lathami (Gray.) Australia. From Mr. J. Gould's Collection.

MIegaughalon rubripes (Quoy \& Gaim.) Celebes Islands. Presented by T. B. Wilson, M. D.

Megarodius tumulus Gould. Australia. From Mr. J. Gould's Collection.

“ Rubripes Temm. Timor Asia. From Mons. Des Murs' Collection.

" Reinwardti Wagl. Celebes. From the same.

Leiron ocellata Gould. Australia. From Mr. J. Gould's Collection.

\section{Family PHASIANID E.}

Pavo cristatus Linn. India. From Mons. Des Murs' Collection.

$\begin{array}{lll}\text { "6 artrous (Linn.) Japan. } & \text { From the same. } \\ \text { From the same. }\end{array}$

Argus giganteus Temm. Japan. From the same.

Phasianus colchicus Linn. France. From the same.

"6 Torquatus Gmel. France. From the same.

Tilaumala picta (Linn.) Asia. From the same.

Gat.lophasis nyctexierus (Linn.) Asia. From the same.

" Leuconelanos (Lath.) Bengal. From the same.

Galtrs Banisiva Temm. India. From the same.

" Domesticus. France. From the same.

Gallus domesticus Var. russicus. France. From the same.
"6 "6 Nanus. France. From the same.
"6 "6 GREeNLandicus. France. From the same.
"6 "6 "6agopus. France. From the same.
6 "6 "6omeranus. France. From the.same.

" Lanatus (Linn.) Java. From the same.

" varius (Shaw.) Java. From the same.

" sonnerati Temm. Asia. From the same.

" Ecaudatis (Linn.) France. From the same.

" Gigaxtevs Temm. Java. From the same.

Meleagris gallopavo Linn. United States. From the same.

6 66 France. From the same.

Nivitida Meteagris Linn. Africa. From the same.

6" 6 France. From the same. 
Tempaogallus catcasicus (Pall.) Asia. Preserted by Mr. Edwatd
Wilson.

\section{Family TETRAONID $Æ$.}

Francolinus Le Vaillanti (Valene). Africa. From M. Des Murs' Collection. "6 Afer (Lath). Africa. From the same.

" capensis (Gmel.) Africa. From the same.

" Nudicollis (Gmel.) Africa. From the same.

" clamator (Temm.) Africa. From the same.

Perdrix cinerea (Linn.) France. From the same.

Coturnix communis Bonn. France. From the same.

" " $"$ Africa. From the same.

" Australis (Lath.) Australia. From Mr. J. Gould's Collection.

6 Pectoralis Gould. Australia. From the same.

" Diemenexsis (Gould.) Australia. From the same.

" Chinensis (Linn.) Java. Presented by Mons. J. Verreaux.

“ Coromandelica (Gmel.) India. Presented by Mr. E. Wilson.

Caccabis rufa (Linn.) France. From Mons. Des. Murs' Collection.

" " "Spain. From the same.

"G GRAECA (Briss.) Switzerland. From the same.

"6 PETrosa (Lath.) France. From the same.

" " 6 Africa. From the same.

" chuкar (Gray.) India. Presented by Mr. E. Wilson.

Turnix rugnax (Temm.) Java. From Mons. Des Murs' Collection.

" varius (Lath.) Australia. From Mr. J. Gould's Collection.

" velox Gould. Australia. From the same.

" Pyrrhothorax Gould. Australia. From the same.

" Castanotus Gould. Australia. From the same.

" scintillans (Gould.) Australia. From the same.

Odontophorus dentatus (Temm.) Brazil. From Mons. Des Murs' Collection.

Ortyx virginianus (Linn.) Pennsylvania. From the same.

" Nigrogularis Gould. Yucatan. Presented by T. B. Wilson, M. D.

Calitpepla Californica (Lath.) California. Presented by A. L. Heermann, M. D.

Tetrao urogallus Linn. Spanish Pyrenees. From Mons. Des Murs' Collection.

"6 myвrdus Linn. Switzerland. From the same.

" TETRIX Linn. Switzerland. From the same.

"S obscurus Say. Rocky Mountains. From the same.

" Canadensis Linn. Nova Scotia. Presented by T. B. Wilson, M. D.

" Phasianeluus Iinn. Missouri. From the same.

6 curido Linn. Illinois. Presented by A. L. Heermann, M. D.

Bonasa timbellus (Linn.) Pennsylvania. Presented by Mr. S. Ashmead.

" sylvesrris Brehm. Switzerland. From Mons. Des Murs' Collection.

Lagopus scoticus (Lath.) Scotland. From the same.

" albus (Gmel.) Russia. From the same.

" Murus Leach. Switzerland. From the same.

"6 Islandorum Faber. Iceland. From the same.

Pterocles alchata (Linn.) French Pyrenees. From the same.

" Arevarius (Pall.) Spain. From the same.

"6 Fasciatus (Scop.) Africa. From the same.

"6 Exustus Temm. Syria in Asia. From the same. 
VI. Family TINAMIDA.

Tinanus tao (Temm.) Brazil. From Mons. Des Murs' Collection. " Mrajor (Linn.) Brazil. From the same.

"v vernictiatrs (Temm.) Mexico. From the same. •

" cromers (Gmel.) Brazil. From the same.

" Noctivagus Pr. Max. Brazil. From the same.

"Vafiegatus (Gmel.) Brazil. From the same.

" uxdelatus Temm. Peru. From the same.

$\therefore$ sovi (Gmel.) Brazil. From the same.

"Tatapae Temm. Chili. From the same.

Nothura Boraquira (Spix.) Rio Janiero. From the same.

" Mryor (Spix.) Brazil. From the same.

" visi ('Temm.) Paraguay. From the same.

" maculosa (Temm.) Brazil. From the same.

Ruycuotus rufescexs (Temm.) Buenos Ayres. From the same.

\section{6th Order STRUTHIONES.}

\section{Family STRUTHIONIDÆ.}

Strutho Camelus Linn. Africa. From M. Des Murs' Collection.

Rifa anericaxa Lath. Brazil. From the same.

"DArwinir Gould. Brazil. From the same.

Dronaits Nove Holdande (Lath.) Australia. From Mr. J. Gould's Collection.

Casaries exu Lath. Ind. Archipelago. From M. Des Murs' Collection.

Apteryx australis Shaw. Australia. Presented by 'T. B. Wilson, M.D.

Otis tarda Linn. France. From M. Des Murs' Collection.

"Tetrax Linn. France. From the same.

Eupodotis Edwardsi (Gray.) India. Presented by Mr. E. Wilson.

" australis (Gray.) Australia. From Mr. J. Gould's Collection.

“ Denifani (Childr.) Africa. From M. Des Murs' Collection.

$\because \quad$ Caffra (Licht.) Africa. From the same.

"Ludwigi (Rupp.) Africa. From the same.

"VIgorsil (A.Smith.) Africa. From the same.

"6 uxdulata (Jacq.) Africa. From the same.

" aurita (Lath.) Bombay. From the same.

\section{7th Order GRALLE.}

\section{Family CHARADRIDE.}

(Edicnemus crefitans Temm. France. From M. Des Murs' Collection.

" "6 Africa. From the same.

"6 Maculosus Temm. Africa. From the same.

"6 bistriatus (Wagl.) From the same.

" graldarius (Lath.) Australia. From Mr.J. Gould's Collection.

Esacus magnirostris (Geoff.) Australia. From the same.

Pluvianus Egyptius (Linn.) Egypt. From M. Des Murs' Collection.

Cursorius coronandelicus (Gmel.) Asia. From the same.

"6 Bicinctus Temm. Africa. From the same.

Giateola pratincola (Linn.) France. From the same. 


\section{7}

Vanellus cristatus Meyer. France. From MI. Des Murs' Collection.

" " " Laponia. From the same.

6 "6ngland. From the same.

" Cayennemsis (Gmel.) Paraguay. From the same.

Lobivanel.tus personata Gould. Australia. From Mr. J. Gould's Collection.

Hoplopterus spinosus (Linn.) Asia. From Mt. Des Murs' Collection.

" PERsicus (Bonn.) Africa. From the same.

"6 coronatus (Bodd.) Africa. Presented by Mr. E. Wilson.

Cimaradrius pluvialis Linn. France. From M. Des Murs' Collection.

" Morinellus Linn. France. From the same.

" vociferus Linn. Maryland. Presented by A. L. Heermann, M.D.

"6 "6uba. From M. La Sagra's Collection.

“ hiatactla Linn. France. From M. Des Murs' Collection.

" Curovicus Beseke. France. From the same.

" cavtianus Lath. France. From the same.

" Bicinctus Jard. and Selby. Australia. From the same.

" RUfictpilur's Temm. Australia. From Mr. J. Gould's Collection.

" collaris Vieill. Buenos Ayres. From M. Des Murs' Collection.

"Wilsonius Ord. N. Jersey. Presented by A. L. Heermann, M. D.

6 Melodus Ord. N. Jersey. From the same.

" SeMraliatus Kanp. Labrador. From M. Des Murs' Collection.

“ xanthocheilus Wagl. N. S. Wales. Presented by M. J. Verreaux.

Hematopus ostralegus Linn. France. From M. Des Murs' Collection.

" falliatus Temm. N. Jersey. Presented by A. L. Heermann, M.D.

"6 Niger Cuv. Australia. From M. Des Murs' Collection.

i6 Capexsis Licht. Africa. From the same.

s6 Fuliginosus Gould. Australia. From Mr. J. Gould's Collection.

6 Longrostris Vieill. Australia. From the same.

Cinclus interpres (Linn.) Germany. From Mr. Des Murs' Collection.

\section{Family ARDEID瓜.}

Psofila crepitans Linn. Amazon River. Presented by Mr. W. H. Edwards.

Grus cinerea Bechst. Germany. From M. Des Murs' Collection.

"Carunculata (Gmel.) Africa. From the same.

" antigone (Linn.) Asia. From the same.

"Canadensis (Linn.) Wisconsin. Presented by A. L. Heermann, M. D.

" australinisa Gould. Australia. From Mr. J. Gould's Collection.

Scops virgo (Linn.) Africa. From M. Des Murs' Collection.

"6 paradisea (Licht.) Africa. From the same.

Eurypya meltas (Pall.) Paraguay. From the same.

Ardea cinerea Linn. France. From the same.

"6 Melanocephala Childr. and Vig. Africa. From the same.

" herodias Linn. Pennsylvania. From the same.

66 "6 Amazon River. Presented by Mr. W. H. Edwards.

"6 purpurea Linn. England. Presented by T. B. Wilson, M. D.

"6 PAcifica Lath. Australia. From Mr. J. Gould's Collection.

" Alba Gmel. Europe. Presented by Mr. F. Wilson.

" EGRETTA Gmel. N. Jersey. Presented by S. W. Wondhouse, M. D.

"6 "6 Amazon River. Presented by Mr. W. H. Edwards.

"6 6 France. From M. Des Murs' Collection.

"G Garzetta Linn. France. From the same. 
Amer candidissma Gmel. N. Jersey. From M. Des Murs' Collection.

" rufa Bodd. Florida. Presented by Mr. J. J. Audubon.

"S Peali Pr. Bonap. Florida. From M. Des MLurs' Collection.

" Leucogaster Gmel. Florida. Presented by A. L. Heermann, M. D.

" Corulea Linn. Florida. From the same.

" KOV. HOLLANDis Lath. Australia. From Mr. J. Gould's Collection.

" Jugularis Forst. Australia. From the same.

" comata Pall. Hungary. From M. Des Murs' Collection.

" minuta Linn. France. From the same.

"6 Exilis Gmel. Pennsylvania. Presented by Professor Baird.

" virescens Linn. Pennsylvania. From M. Des Murs' Collection.

"Grisea Bodd. Chili. From the same.

" Flavicoltits Lath. Australia. From Mr. J. Gould's Collection.

" picata Gould. Australia. From the same.

" Levcoptera Bodd. Australia. From the same.

" coromanda Bodd. Europe. From M. Des Murs' Collection.

Botaurus stellaris (Linn.) France. From the same.

"sextigivosus Mont. California. Presented by A. L. Heermann, M.D.

" "Wissouri. Presented by G. C. Leib, M. D.

Nicticorax n vius (Bodd.) Florida. Presented by A. L. Heermann, M. D. " Caledonicus (Gmel.) Australia. From Mr. J. Gould's Collection. "violaceus (Lina.) Florida. Presented by T. B. Wilson, M. D. "Griseus (Linn.) Europe. From the same.

Platalea leucorodia Linn. Germany. From Mr. Des Murs' Collection.

" AJAJA Linn. Amazon River. Presented by Mr. W. H. Edwards.

Ciconia alba Briss. Germany. From M. Des Murs' Collection.

"6 NIGRa Bechst. Russia. From the same.

'Tantalus loculator Linn. Amazon River. Presented by Mr. W. H. Edwards.

Inis rubra (Linn.) Brazil. From M. Des Murs' Collection.

" Falcineldus (Linn.) Hungary. From the same.

" Aliza (Linn.) Florida. Presented by Mr. J. J. Aurubon.

\section{Family SCOLOPACIDÆ.}

Numentus arcuatus (Linn.) Germany. From M. Des Murs' Collection. is pheopus (Linn.) Germany. From the same.

Limosa exgocephata (Linn.) England. Presented by T. B. Wilson, M. D. " Laproxica (Linn.) Holland. From M. Des Murs' Collection.

Totanus calidors (Linn.) France. From the same.

" ochropus (Linn.) Savoy. From the same.

"s Flavipes (Gmel.) Pennsylvania. Presented by Mr. S. Ashmead.

" Semipalmatus (Gmel.) France. From M. Des Murs' Collection.

" "N. Jersey, Presented by Mr. John Krider.

"c Chlorofygius Vieill. Pennsylvania. Presented by A. I. Heermanu, M. D.

Tringoides hypoleuca (Linn.) France. From M. Des Murs' Collection.

" Macularia (Linn.) Maryland. Presented by A. L. Heermam, M.D.

"Bartramius (Wils.) Missouri. Presented by Wm. Gambel, M. D.

Rectrvirostra avocetta Linn. France. From M. Des Murs' Collection.

" a.rericana Gmel. Rocky Mits. Presented by A. L. Heermann, M. D.

Hrimantopus Candidus Bonn. N. Africa. Presented by T. B. Wilson, M. D. 
Prilonacius pugrax (Linn.) Germany. From M. Des Murs' Collection.

Tringa maritima Brün. Iceland. From the same.

" RUfescexs Vieill, Labrador. From the same.

" cryclus Linn. Sweden. From the same.

" scurvari Brehm. Labrador. From the same.

" canurus Linn. Iceland. From the same.

Calidris aremiria (Linn.) Norway. From the same.

Galitiago major (Gmel.) Africa. From the same.

" MEDIA Steph. France. From the same.

"6 Gallintla (Linn.) France. From the same.

" Wilsoxin (Temm.) Nova Scotia. Presented by T. B. Wilson, M.D.

" uxdilata (Bodd.) Brazil. From M. Des Murs' Collection.

Scolopax rusticola Linn. France. From the same.

Pinloheli mivor (Gmel.) Pennsylvania. From the same.

Rhyncilea capensis (Linn.) Africa. From the same.

Pralaropis rulicarius (Linn.) Iceland. From the same.

" hYPerdoreus (Linn.) Iceland. From the same.

IV. Family PALAMEDEIDE.

Para jacana Linn. Brazil. From M. Des Murs' Collection.

Palamedea connuta Linn. Brazil. From the same.

Chauna chavaria (Linn.) Paraguay. From the same.

\section{Family RALLIDÆ.}

Rallus aquaticus Linn. France. From M. Des Murs' Collection.

" vikginianus Linn. N. Jersey. Presented by Mr. J. Krider.

" crepitans Gmel. N. Jersey. From the same.

" Eregars Aud. S. Carolina. Presented by T. B. Wilson, M. D.

" superciliosus Swains. Sumatra. From M. Des Murs' Collection.

" phimpensis Linn. Australia. From Mr. J. Gould's Collection.

" pectoralis Cuv. Australia. From the same.

Aranus scolopaceus (Gmel.) Paraguay. From M. Des Murs' Collection.

Ortygonetra crex (Gmel.) France. From the same.

" carolina (Linn.) Massachusetts. Presented by T. B. Wilson, M. D.

" rorzana (Linn.) France. From M. Des Murs' Collection.

" Jamaicersis (Gmel.) N. Jersey. Presented by Mr. S. Ashmead.

" affinis G. R. Gray. Australia. From M. Des Murs' Collection.

* Mivuta (Pall.) Spain. From the same.

Aramides gigas (Spix.) Brazil. From the same.

"6 Maculatus (Bodd.) Paraguay. From the same.

" C.esius (Spix.) Paraguay. From the same.

66 CaYenxensis (Gmel.) Cayenne. From the same.

Eumaegornis castaneoventris Gould. Australia. From Mr. J. Gould's Collection.

Coretherra rubigixosa (Temm.) Sumatra. From M. Des Murs' Collection. "G Fasciata (Raffl.) Java. From the same.

" Fusca (Linn.) Sumatra. From the same.

Ponpiyrio martinica (Linn.) Louisiana. From the same. 
Porrimeio meranocephalus (Gmel.) Cayana. From M. Des Murs' Collection.

" purpureus (Gmel.) Brazil. From the same.

Tribosyx Mortieri Dubus. Australia. From Mr. J. Gould's Collection.

"6 Vevtralis (Gould.) Australia. From the same.

Gallinrta cirloropts (Linn.) France. From M. Des Murs' Collection.

" pilexicura (Penn.) Java. From the same.

" Crassirostris (Gray.) Brazil. From the same.

"s Galeata Pr. Max. Florida. Presented by J. J. Audubon.

Fulica atra Linn. France. From M. Des Murs' Collection.

"G Cristata Gmel. Africa. From the same.

" armillata Vieill. Chili. From the same.

"Gigantea Eyd. et Souley. Peru. From the same.

"G americaya Gmel. California. Presented by A. L. Heermann, M. D.

\section{8th Order ANSERES.}

I. Family ANATID E.

Puquicopterus antiquorum Temm. France. From M. Des Murs' Collection. "6 Barbary. Presented by T. B. Wilson, M. D.

Plectropteres gamiensis (Linn.) Africa. From M. Des Murs' Collection.

Chenalotex egrptiaces (Linn.) Egypt. From the same.

Cereopsis nove holdandie Lath. Australia. From Mr. J. Gould's Collection.

Anser ferus Gesn. France. From M. Des Murs' Collection.

"SEgetum (Gmel.) Norway. From the same.

" brachy

" ERYThrores (Linn.) Russia. From the same.

"6ygyoldes (Linn.) China. From the same.

Bernicla canadensis (Linn.) Missouri. From the same.

Nettarus rulcuejus Gould. Australia. From Mr. J. Gould's Collection.

Cygnus olor (Gmel.) France. From M. Des Murs' Collection.

" " England. Presented by T. B. Wilson, M. D.

". Ferus Ray. Northern Europe. From M. Des Murs' Collection.

" NIGRICorlis (Gmel.). Chili. From the same.

" ATrata (Lath.) Austratia. From Mr. J. Gould's Collection.

Dendrocygna arcuata (Cuv.) Australia. From the same.

"6 " Nepaul, India. Presented by Mr. E. Wilson.

“ viduata (Linn.) S. America, From M. Des Mlurs' Collection.

Tadorna vulfanser Flem. England. From the same.

"6 "6rance. From the same.

" Nævosa (Gould.) Australia. From Mr. J. Gould's Collection.

Aix sponsa (Linn.) Virginia. From M. Des Murs' Collection.

"6 6) Cuba. From MI. La Sagra's Collection.

"G Galericulata (Linn.) China. Presented by Mr. E. Wilson.

Mareca penelope (Linn.) Northern Europe. From M. Des Murs' Collection.

Dafila acuta (Linn.) Iceland. From the same.

Anas moschas Linn. France. From the same.

" " " $\quad$ Nova Scotia. Presented by T. B. Wilson, M. D.

" "6 California. Presented by A. L. Heermann, M. D.

" superciliosa Gmel. Australia. From Mr. J. Gould's Collection. 
Axas obscura Gmel. N. Jersey. Presented by Mr. S. Ashmead.

66 punctata Burch. Australia. From Mr. J. Gould's Collection.

Querqueduza crecca (Linn.) Holland. From M. Des Murs' Collection.

"6 Ipecuturi (Vieill.) Rio de la Plata. From the same.

Pterocyanea crrcia (Linn.) Hungary. From the same.

"s Discons (Linn.) Missouri. Presented by A. L. Heermann, M. D.

6: caruleata (Licht.) California. From the same.

Chauleqasmus strepera (Linn.) Holland. From M. Des Murs' Collection.

Situlua clypeata (Linn.) Rocky Mis. Presented by A. L. Heermann, M.D. "6 "Holland. From M. Des Murs' Collection.

Caimina moschata (Linn.) Brazil. From the same.

Bravta Rufiva (Pall.) Sicily. From the same.

66 "Lucknow. Presented by T.B. Wilson, M.D.

Fultgula cristata (Ray.) Iceland. From M. Des Murs' Collection.

"6 Marila (Linn.) Iceland. From the sarne.

Nyroca ferina (Linn.) Saxony. From the same.

"6 LeUCopthalma (Bechst.) Saxony. From the same.

Hydridus N.Valesneria (Wils.) et Anas boschas Linn. Pennsylvania. Pre. sented by Professor Baird.

Clingula glaucion (Linn.) Northern Europe. From M. Des Murs' Collection.

"ISIANDICA (Gmel.) Iceland. From the same.

$\therefore$ Histrionica (Linn.) Iceland. From the same.

Harelda glacialis (Linn.) Sweden. From the same.

Somateria molissima (Linn.) Spitzberg. From the same.

OIDfaira NIGRA (Linn.) Iceland. From the same.

"6 velavetiva Cassin. Labrador. From the same.

6. PERspicillata (Linn.) Labrador. From the same.

Biziura lobata (Shaw.) Australia. From Mr. J. Gould's Collection.

Frismatura leucocephala (Scops.) Europe. From M. Des Murs' Collection. 66 australis (Gould.) Australia. From Mr. J. Gould's Collection.

Mergus castor Linn. Norway. From M. Des Murs' Collection. "6 Serrator Linn. Sweden: From the same.

"6 : Bay of Fundy. Presented by T. B. Wilson, M. D.

"6 cuculcatus Linn. Labrador. From M. Des Murs' Collection.

II. Family COLYMBID

Cozrmbus glactalis Linn. Northern Europe. Frum M. Des Murs' Collection. "6 Arcricus Linn. Northern Europe. Presented by Mr. E. Wilson.

66. Septevtrionalis Linn. Iceland. From M. Des Murs' Collection.

Podiceps cristatus (Linn.) Northern Europe. From the same.

"6 Arcticus Boie. Iceland. From the same.

"6 Grisegend (Bodd.) Northern Europe. From the same.

$66 \quad$ Africa. From the same.

"6 Atritus (Linn.) Europe. Presented by T. B. Wilson, M. D.

66 Mivor (Gmel.) Europe. From M. Des Murs' Collection.

“ connutus (Gmel.) Germany. From the same.

"6 Philippensis (Bonn.) Java. From the same.

66 Nove nollandie Steph. Australia. From Mr. J. Gould's Collection. 
Podichibrs caroninersis (Lath.) Missouri. Presented by A. L. Heermann, M. D.

From M. La Sagra's Collection.

\section{Family ALCID $\approx$.}

Alca mirexnis Linn. Aretic Furope. From MI. Des Murs' Collection.

"6 Torpa Linn. Iceland. From the same.

" " Labrador. From the same.

Fratercula Ghaclatis (Leach.) Northern Europe. From the same.

" " Labrador. Presented by T. B. Wilson, M.1).

$\because \quad$ arctica (Linn.) Normandy. From M. Des Murs' Collection.

"G cirniata (Gmel.) California. Presented by A. L. Heermann, II. D.

Sprieniscrs denrersus (Linn.) Cape Good Hope. From M. Des Murs' Collection.

" Magfilanicus (Forst.) Cape Good Hope. Presented by Mr. E. Wilson.

Eudyptes aytarctica (Forst.) Antarctic Ocean. From M. Des Mrurs' Collection.

" cirrysocosie (Forst.) S. African Coast. From the same.

" Torquata (Forst.) Antarctic Ocean. From the same.

" Mrvor (Forst.) S. A frican Coast. From the same.

"6 Australia. From Mr. J. Gould's Collection.

Aptevonytes Forsteri G. R. Gray. Southern Ocean. From M. Des Murs? Collection.

URIA GRYLLE (Linn.) Arctic Europe. From the same.
"6 "6 6 Labrador. Presented by T. B. Wilson, M. D.
California. Presented by A. L. Heermann, M. D.
'Mandtil Licht. Aretic Europe. From Mi. Des Murs' Collection.
" Troile (Linn.) N. Europe. From the same.
"6 "Troile (Linn.) Newfoundland. From the same.

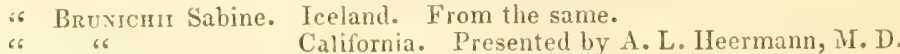
"6 rivgria Brïnn. Iceland. From M. Des Murs' Collection.

\section{Family PROCELLARID E.}

Puffints major Faber. Sardinia. From M. Des Murs' Collection.

:" Sphenures Gould. Australia. From the same.

" angloria Ray. England. From the same.

“ obscurus (Gmel.) Europe. Presented by T. B. Wilson, M. D.

"6 chlororhyxchus Less. Australia. From Mr. J. Gould's Collection.

"6 Carnipes Gould. Anstralia. From the same.

" Brevicaudes Gould. Australia. From the same.

“Fouinoctialis (Linn.) Island of Bourbon. From M. Des Murs'

Collection.

Timalassidroma peragica (Linn.) Sardinia. From the same.

" Leacil (Temm.) France. From the same.

"6 Bay of Fundy. Presented by T. B. Wilson, M. D.

"Bchwerir (Jard. and Selby.) Madeira. From the same.

" Mariva (Linn.) Australia. From Mr. J. Gould's Collection.

Proceicaria gigantea Gmel. Antaretic Circle. From M. Des Murs' Collection.

"6 Glactalis Linn. Northern Europe. From the same.

65 66 Malouine Islands. From the same.

"solandri Gonld. Australia. From Mr. J. Gould's Collection. 
Prion vittata (Gmel.) Antarctic Ocean. From Mr. J. Gould's Collection.

Diomedea exulans Linn. Van Diemen's Land. From the same.
"6 S. African Coast. From M. Des Murs' Collection.
"6 Cauta Gould. Australia. From Mr. J. Gould's Collection.
"6 Cillororiny nera Gmel. Cape Horn. From M. Des Murs' Collection.
" Fuliginosa Gmel. Gape Horn. From the same.

\section{Family LARIDæ.}

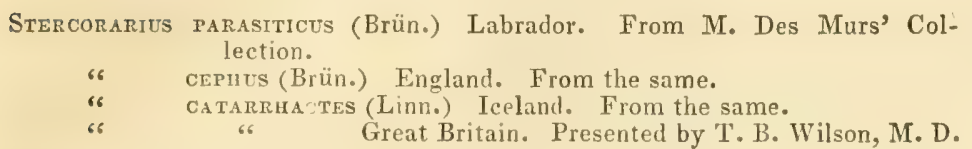

Rhodostetuia Rossi (Sabine.) Aretic Circle. From M. Des Murs' Collection.

Larus glaucus Brünn. Spitzberg. From the same.

6 G6 Greenland. From the same.

" Marinus Linn. Europe. From the same.

6 " Labrador. From the same.

"6 LEucopterus Faber. France. From the same.

6. 6 Labrador. Presented by T. B. Wilson, M. D.

" argentatus Brünn. France. From M. Des Murs' Collection.

" " Lalirador. From the same.

" occidentalis Aud. California. Presented by A. I. Heermann, M. D.

"6 Fuscus Linn. France. From M. Des Murs' Collection.

"6 Pacificus Lath. Australia. From Mr. J. Gould's Collection.

" Nove Hollande Steph. Australia. From the same.

“ Crassirostris Vieill. Van Diemen's Land. From Mons. Des Murs'

Collection.

" ICHтнетus Pall. Eastern Europe. From the same.

"CANus Linn. Europe. From the same.

"6 zonorhynchus Rich. and Swains. Labrador. Presented by T. B. Wilson, M. D.

“ Audounin Payr. Poland. From M. Des Murs' Collection.

" Ridibundus Linn. France. From the same.

" Capistratus Pr. Bonap. Europe. From the same.

“ atricillus Linn. N. Jersey. Presented by Wm. Gambel, M. D.

" " 6 Europe. From M. Des Murs' Collection.

" Leucopthalmus Licht. Southern France. From the same.

" Gelastes Licht. Southern France. From the same.

" cirrocepiialus Vieill. Buenos Ayres. From the same.

Xema SAbinei Leach. Furope. From the same.

Rissa tridactyla (Lath.) England. Presented by T. B. Wilson, M. D. "6 " Labrador. From the same.

Ruyncops nigra Linn. N. Jersey. Presented by Mr. J. Krider.

" Melanura Swains. S. America. Frum M. Des Murs' Collection.

"6 alibrostris Licht. Africa. From the same.

Sterna caspia Pall. France. From M. Des Murs' Collection.

"6 Pelicanoides Vig. Australia. From Mr. J. Gould's Collection.

" Porfocercus Gould. Anstralia. From the same.

"6 AFFinis Rupp. Java. From M. Des Miurs' Collection.

“6 Cayennensis Gmel. Florida. Presented by A. L. Heermann, M. D.

"6 "Amazon River. Presented by Mr. W. H. Edwards.

“ anglica Mont. France. From M. Des Murs' Collection.

66 " New Jersey. Presented by Mr. J. Krider. 
Sterna strevuus Gould. Australia. From Mr. J. Gould's Collection.

"6 Fulicinosa Gmel. Australia. From the same.

"6 of Tortugas Islands. Presented by A. L. Heermann, M.D.

6. ranaya Gould. Australia. From Mr.J. Gould's Collection.

"6 acutrelavid Cabot. Florida. Presented by A. L. Heermann, M. D.

"Cantiaca Gmel. Erance. From M. Des Murs' Collection.

": IIRundo Linn. France. From the same.

"6 Africa. From the same.

macroura Naum. Iceland. From the same.

"6 N. Jersey. Presented by A. L. Heermann, M. D.

Wilsonil Pr. Bonap. N. Jersey. From the same.

paradisea Brün. Europe. From M. Des Murs' Collection.

Minuta Linn. France. From the same.

Argentea Pr. Max. New Jersey. Presented by Mr. J. Krider.

melanatichen Temm. Timor, Asia. From M. Des Murs' Collection. sfeculifera Temm. Paraguay, From the same.

NERErs Gould. Australia. From Mr. J. Gould's Collection.

Hydrochelidon hytida (Pall.) France. From M. Des Murs' Collection. "YIGRA (Linn.) France. From the same.

66 Leucoptera (Temm.) Greece. From the same.

Axous stourdus (Lim.) Portugal. From the same.

66 Australia. From Mr. J. Gould's Collection.

66

Tortugas Islands. Presented by A. L. Heermann,M. D.

Melanops Goulu!. Australia. From Mr. J. Gould's Collection.

gracilis Gould. Australia. From the same.

Levcocapiluus Gould. Australia. From the same.

"6 TERETicolis (Lafr.) Australia. From the same.

VI. Family PELICANID E.

Przeton atrereus Linn. Island of Bourbon. From M. Des Murs' Collection.

" Rubricauda Bodd. Australia. From Mr. J. Gould's Collection.

" Candidus (Briss.) Brazil. From M. Des Murs' Collection.

Plotus animga Linn. Florida. Presented by T. B. Wilson, M. D.

" Melanogaster Gmel. Brazil. From M. Des Murs' Collection.

"6 congensis Leach. Africa. From the same.

Sula bassana (Linn.) Holland. From the same.

" " Labrador. Presented by E. Harris, M. D.

" Carensis Licht. Africa. From M. Des Murs' Collection.

" cyanops Sunder. Australia. From Mr. J. Gould's Collection.

" personata Gould. Anstralia. From the same.

"6 Piscator (Linn.) Australia. From the same.

" Fusca Vieill. Australian Coast. From the same.

Graculus carbo (Linn.) Holland. From M. Des Murs' Collection.

"Sinevsis (Shaw.) Antarctic America. From the same.

" Carboldes (Gould.) Australia. From Mr. J. Gould's Collection.

"6ristatus (Fabr.) France. From M. Des Murs' Collection.

" Linnei Gray. Africa. From the same.

"Desiraresti (Payro) Corsica. From the same.

" Dilophus (Vieill.) Labrador. Presented by T. B. Wilson, M. D.

66 Floridianes (Aud.) Florida. Presented by A. L. Heermann, M. D. 
Graculus Townsendis (Aud.) California. Presented by A. L. Heermann, M. D.

"v violaceus (Gmel.) California. From the same.

“ GaimardiI (Garn.) Chili. From M. Des Murs' Collection.

6 LeUcogaster (Gould.) Australia. From Mr. J. Gould's Collection.

“ pygmeus (Pall.) Europe. From M. Des Murs' Collection.

" Melanoleucus (Vieill.) Australia. From Mr. J. Gould's Collection.

“ Africanus (Gmel.) Afriaa.' Presented by T. B. Wilson, M. D.

Pelecanus onocrotalus (Linn.) Africa. From M. Des Murs' Collection.

6. conspicillatus Temm. Australia. From Mr. J. Gould's Collection.

“ Fusces Linn. Florida. Presented by A. L. Heermann, M.D.

" " Texas. From M. Des Murs' Collection.

Atagen aquila (Linn.) Island of Ascension. From the same.

The foregoing catalogue of birds' eggs embraces 1323 determined species of 493 genera. Of these, 835 species, derived from all parts of the world, formed the celebrated and valuable collection of Mons. O. Des Murs, of Paris. The Australian species, numbering 246, accompanied the splendid collection of birds from that country, made by the distinguished ornithologist Mr. John Gould, of London, and which is now in the Museum of the Academy. The Cuba species belonged to the collection of the well known naturalist M. Ramon de la Sagra, and were presented by Mr. E. Wilson.

The whole of the two first collections were purchased by Dr. T. B. Wilson, and, with his usual liberality, presented to the Academy. The remainder consists of species presented by several individuals whose names are attached to their donations throughout the catalogue. Of undetermined eggs in the collections of M. Des Murs and Mr. Gould, there are 197 species.

\section{CORRECTIONS.}

Page 4. For leucostemus read leucosternus.

Page 11. Add Lawrence after Conirostum ornatum. 


\section{INDEX FAMILIARUM.}

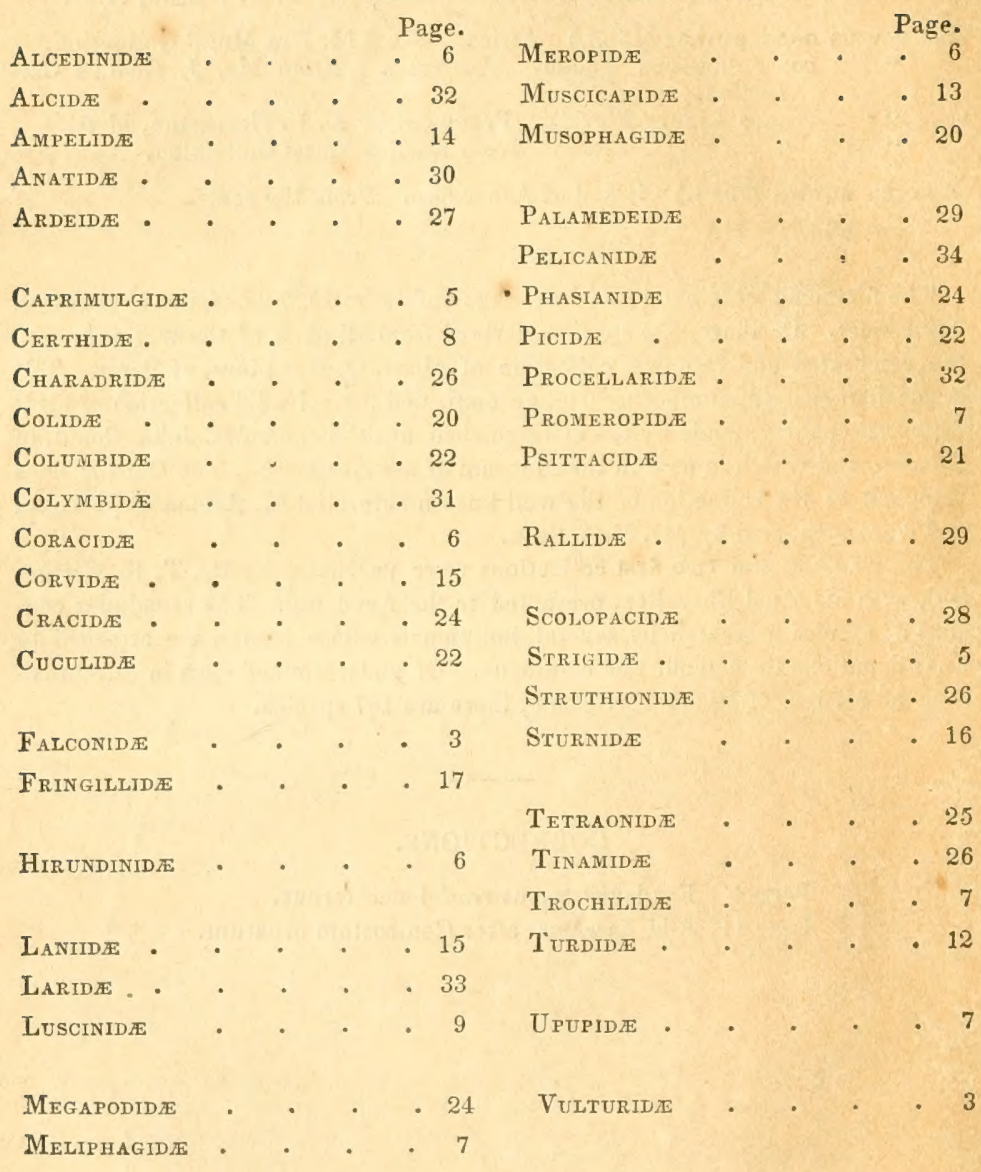



